\title{
Cycle spaces in topological spaces
}

\author{
Antoine Vella And R. Bruce Richter \\ University of Waterloo
}

10 December 2007

\begin{abstract}
We develop a general model of edge spaces in order to generalize, unify, and simplify previous work on cycle spaces of infinite graphs. We give simple topological criteria to show that the fundamental cycles of a (generalization of a) spanning tree generate the cycle space in a connected, compact, weakly Hausdorff edge space. Furthermore, in such a space, the orthogonal complement of the bond space is the cycle space. This work unifies the two different notions of cycle space as introduced by Diestel and Kühn (Combinatorica 24 (2004), 68-89 and Europ. J. Combin. 25 (2004), 835-862) and by Bonnington and Richter (J. Graph Theory 44 (2003), 132-147).
\end{abstract}

\section{Introduction}

In [1], Bonnington and Richter introduce the cycle space of a locally finite graph as the set of edge-sets of subgraphs in which every vertex has even degree. Motivated by this work, Richter asked when the cycle space of an infinite graph is generated by fundamental cycles. In response, Diestel and Kühn [8] introduced a different notion of cycle space of a locally finite graph.

In an infinite graph $G$, two rays (or 1-way infinite paths) in $G$ are equivalent if, for every finite set $W$ of vertices, the tails of the two rays are in the same component of $G-W$. An end of $G$ is an equivalence class of rays. The Freudenthal compactification $\mathbf{F}(G)$ is the topological space obtained from the 1-dimensional cell complex on $G$ by adding a point for each of the ends of $G$. A basic neighbourhood of an end $\omega$ is the topological component of $G-W$ (for a finite subset $W$ of $V(G)$ ) containing rays in $\omega$, plus all the other ends with rays in this same component. If $G$ is locally finite, then $\mathbf{F}(G)$ is compact, justifying the name ([6] Prop. 8.5.1).

In [8], Diestel and Kühn consider a locally finite graph $G$ and focus on homeomorphs of circles in $\mathbf{F}(G)$. For example, if two rays in the same end have a common origin but are otherwise disjoint, then, in $\mathbf{F}(G)$, the union of the two rays plus their common end point is a homeomorph of a circle. If $C_{1}, C_{2}, \ldots$ is a possibly infinite sequence of edge-sets of homeomorphs of circles such that each edge is in only finitely many of the $C_{i}$, then $\sum_{i \geq 1} E\left(C_{i}\right)$ can be defined to be the 
symmetric difference of all the $C_{i}$ - an edge is in $\sum_{i \geq 1} C_{i}$ if and only if it is in an odd number of the $C_{i}$. The cycle space of Diestel and Kühn is the set of all such sums.

Intriguingly, one can view the cycle space of Bonnington and Richter as the Diestel-Kühn cycle space of the Alexandroff, or 1-point, compactification $\mathbf{A}(G)$ of the locally finite graph $G$. That is, if $C_{1}, C_{2}, \ldots$ is a possibly infinite sequence of edge-sets of homeomorphs of circles in $\mathbf{A}(G)$ such that every edge is in finitely many of the $C_{i}$, then $\sum_{i \geq 1} E\left(C_{i}\right)$ has each vertex incident with an even number of its edges. Conversely, the edge-set of any subgraph having even degree at every vertex can be expressed as such a sum (this will follow from the results in this work and was also proved in [1]). This connection was a principal motivation for our researches.

With these two particular cases in mind, one realizes that there are many other candidate spaces. Any compactification of a locally finite graph, such as the closure of an embedding in a compact surface (the sphere in particular was considered in [1]), has its own cycle space. An intriguing possibility, whose exploration is begun in [3], is to show that these cycle spaces have natural relationships: if $f: C_{1} \rightarrow C_{2}$ is a continuous surjection of one compactification $C_{1}$ of a locally finite graph $G$ to another compactification $C_{2}$ of $G$, then the cycle space of $C_{1}$ is a subspace of the cycle space of $C_{2}$. These connections merit further study, since it is not yet clear what a basis for the quotient space looks like this was one of the questions studied in [1] for graphs embedded in the sphere, but the general case is wide open.

We adopt a topologically biased approach to cycle space questions. In Section 2 , we introduce the notion of an edge space and in Section 3 describe topological properties of the edge space that are used to prove the cycle space is the space orthogonal to the bond space. Also in this section is the fact that elements of the cycle space partition into cycles. In Section 4, we show that the fundamental cycles of any (generalization of a) spanning tree generate the cycle space. In Section 5, we shall describe a general quotient construction which turns "spaces with edges" into edge spaces, i.e., so that the edges are singletons. In cases of interest, this quotient preserves the cycle space. We then show in Section 6 that our results imply the Diestel-Kühn results related to cycle spaces in a more general setting than locally finite graphs. Thus, a substantial goal of this work is realized, namely to show that the reliance on homeomorphs of circles is not necessary. We conclude with a short section explaining how the context may be generalized to allow infinite sets of parallel edges.

Our starting point, from which the theory presented here developed, was that of trying to reconcile graph-connection with topological connection. If $G=$ $(V, E)$ is a graph, then there is a natural edge space (a general definition will follow shortly) ( $V \cup E, E)$ associated with $G$, whereby the basic open sets are the singletons $\{e\}$, for $e \in E$, and the sets $N(v)$, consisting of a vertex $v$ and 
all its incident edges. We call this topology the classical topology. ${ }^{1}$ Note that the classical topology is Alexandroff discrete, that is, the arbitrary intersection of open sets is open (see [12]).

It is an easy exercise to show that $G$ is connected in the graph-theoretic sense if and only if the classical topology on $V \cup E$ yields a space connected in the sense of point-set topology. It will turn out that, for the cycle space theory, it is in some sense only the edges that really matter. This is perhaps not so surprising, in view of the fact that matroid theory successfully generalizes cycle spaces of finite graphs. Thus, we will generalize the topological context even further to emphasize this fact.

We find it interesting that we can deal directly with topologies on graphs in which the edges are taken to be open singletons. The closure of an edge is just the edge and its incident vertices. In this version, the topological spaces are not Hausdorff, but we will be dealing with natural extensions of this property suitable to the context. We believe that the structural simplicity gained in this model for cycle space problems outweighs the small price paid in having to rework a few topological theorems in this new setting.

We now describe the two topological concepts that we need for our main theorems. A topological space $X$ is weakly Hausdorff if, for any two points $x$ and $y$ of $X$, there are open sets $U_{x}$ and $U_{y}$, containing $x$ and $y$, respectively, such that $U_{x} \cap U_{y}$ is finite. If $G$ is a possibly infinite simple graph with the classical topology, then $G$ is weakly Hausdorff but, as soon as some two vertices are adjacent, not Hausdorff.

An edge space $(X, E)$ is a topological space $X$ and a subset $E \subseteq X$ consisting of points $e$ such that $\{e\}$ is open but not closed and the boundary of $\{e\}$ has at most two points. Henceforth we do not always distinguish between a point and the corresponding singleton; in particular we refer to "open" (or "closed") points, and write $X \backslash e$ for $X \backslash\{e\}$. The elements of $E$ are edges and any point of $X \backslash E$ is a vertex. Notice that a vertex need not be a closed singleton, nor need it be isolated from other vertices; indeed, $X \backslash E$ can have non-trivial components. A vertex $v$ is incident with an edge $e$ if $v \in \operatorname{Cl}(e)$. Because $e$ is open and not closed, it has at least one incident vertex.

In the theory of minors of graphs, a graph $H$ is a minor of $G$ if there is a set $\left\{K_{v} \mid v \in V(H)\right\}$ of disjoint connected subgraphs $K_{v}$ of $G$ and an injection $f: E(H) \rightarrow E(G)$ so that, if $e \in E(H)$ has ends $u$ and $v$, then the ends of $f(e)$ are in $K_{u}$ and $K_{v}$. Then $(G, f(E(H)))$ is an edge space that "represents" the graph $H-H$ is obtained by contracting each subgraph $K_{v}$ to single vertex. The edge space version has the nice feature that it does not require actually changing the ambient space (i.e., $G$ ) in order to discuss properties of $H$. We use this idea in Corollary 20.

\footnotetext{
${ }^{1}$ Note that this differs from the usual topology of a one-dimensional cell-complex associated with a graph. It is "classical" in the sense that it strictly preserves the graph-theoretical notion of connectedness.
} 
One of our main results (Corollary 5 and Theorem 18) is the following.

Theorem 1. Let $(X, E)$ be a connected, compact, weakly Hausdorff edge space. There is a minimal connected subset of $X$ containing $X \backslash E$ and the fundamental cycles of such a subset generate the cycle space.

At this point, the reader does not really know what the cycle space is or what a fundamental cycle is, but we hope the idea of what we are aiming for is clear. We point out here, and show in Section 6, that these theorems subsume essentially all the cycle space theory developed by Diestel and Kühn (although not that of later works by Diestel and his students, especially [2], but see also [7]).

This work is based on part of the first author's Ph.D. dissertation written under the supervision of the second author.

\section{Edge Spaces}

In this section we provide some technical background about edge spaces that we will need in this work. The important point that we prove is Theorem 3, which proves the existence of certain minimal connecting sets. This is used to provide "spanning trees" and "fundamental cycles".

One of the advantages of edge spaces is that we do not need to work only with the classical topology. Indeed, Diestel and Kühn make it clear that there are good reasons for considering other possibilities. In particular, they suggest there are legitimate reasons for thinking an end should be a point treated on a par with the vertices and edges of the graph.

Many basic facts from elementary topology about Hausdorff spaces have natural analogues for weakly Hausdorff spaces. For one that is of use to us, we need an additional notion. A topological space $X$ is weakly normal if, for any two disjoint closed sets $C$ and $D$ in $X$, there are open sets $U_{C}$ and $U_{D}$ in $X$, containing $C$ and $D$, respectively, such that $U_{C} \cap U_{D}$ is finite. The following fact is proved in the standard way.

Lemma 2. Let $X$ be a compact, weakly Hausdorff space. Then $X$ is weakly normal.

The following theorem, the main result of this section, gives us our "spanning trees" and "fundamental cycles". This is a variation of a standard fact $[4, \mathrm{Ch}$. 3, Ex. G 9].

Theorem 3. Let $X$ be a connected, compact, weakly Hausdorff space and let $A \subseteq X$. Then there is a minimal closed connected subset $C$ of $X$ such that $A \subseteq C$.

Proof. In order to apply Zorn's Lemma, we let $\mathcal{C}$ be a chain of closed connected subsets of $X$ containing $A$, ordered by inclusion. Let $\widehat{C}=\bigcap \mathcal{C}$. Evidently $\widehat{C}$ is closed and contains $A$, so it suffices to show $\widehat{C}$ is connected.

Suppose to the contrary that $\widehat{C}$ has a separation $\left(K_{1}, K_{2}\right)$. Both $K_{i}$ are closed in $X$. Since $X$ is, by Lemma 2, weakly normal, there are open sets $U_{1}$ and $U_{2}$ 
containing $K_{1}$ and $K_{2}$, respectively, such that $\left|U_{1} \cap U_{2}\right|$ is finite. Because the $K_{i}$ are closed, we may assume that $U_{1} \cap K_{2}$ and $U_{2} \cap K_{1}$ are both empty. This implies that $U_{1} \cap U_{2}$ is disjoint from $\widehat{C}$. Since $U_{1} \cap U_{2}$ is finite, there is a $C_{1} \in \mathcal{C}$ such that $U_{1} \cap U_{2}$ is disjoint from $C_{1}$.

Suppose $C_{1} \nsubseteq U_{1} \cup U_{2}$. For each $x \in C_{1} \backslash\left(U_{1} \cup U_{2}\right), x \notin \hat{C}$, so there is a $C_{x} \in \mathcal{C}$ such that $x \notin C_{x}$. Since $C_{x}$ is closed, there is an open set $U_{x}$ containing $x$ and disjoint from $C_{x}$. The open sets $U_{x}$, for $x \in C_{1} \backslash\left(U_{1} \cup U_{2}\right)$, cover the closed and compact set $C_{1} \backslash\left(U_{1} \cup U_{2}\right)$. Therefore, there is a finite subcover, say $\left\{U_{x} \mid x \in J\right\}$.

For each $x \in J, U_{x}$ is disjoint from $C_{x}$. There are only finitely many such $x$, so that there is a $C_{2} \in \mathcal{C}$ disjoint from all the sets $U_{x}$, for $x \in J$. This implies that $C_{2} \subseteq C_{1}$ and, furthermore, $C_{2}$ is disjoint from $C_{1} \backslash\left(U_{1} \cup U_{2}\right)$. This implies that $C_{2} \subseteq U_{1} \cup U_{2}$.

We conclude that, in every case, there is a $C_{2} \in \mathcal{C}$ such that $C_{2} \subseteq C_{1}$ and $C_{2} \subseteq U_{1} \cup U_{2}$. It follows immediately that $\left(U_{1} \cap C_{2}, U_{2} \cap C_{2}\right)$ is a separation of $C_{2}$, contradicting the fact that $C_{2}$ is connected.

Given edge spaces $(X, E),(Y, F)$, the latter is an edge subspace of the former if $Y$ is a (topological) subspace of $X, F=E \cap Y$ and, for all $e \in F, \mathrm{Cl}_{X}(e) \subseteq Y$. Note that, if $C \subseteq X$ is closed, $(C, E \cap C)$ is an edge subspace of $(X, E)$. Hence we have the following corollaries.

Corollary 4. Let $(A, F)$ be an edge subspace of a connected, compact, weakly Hausdorff edge space $(X, E)$. Then there is a minimal closed, connected edge subspace $(C, G)$ of $X$ such that $(A, F)$ is an edge subspace of $(C, G)$.

Corollary 5. Let $(X, E)$ be a connected, compact, weakly Hausdorff edge space. Then there is a minimal subset $E^{\prime}$ of $E$ such that $(X \backslash E) \cup E^{\prime}$ is connected.

The set $(X \backslash E) \cup E^{\prime}$ is a generalization of a classical spanning tree; it includes all the non-edges of $X$ and it keeps just enough edges to retain connection. We shall refer to the sets $(X \backslash E) \cup E^{\prime}$ whose existence is guaranteed by Corollary 5 as minimal connected spanning sets for $(X, E)$.

In fact, we can prove Corollary 5 for weakly normal edge spaces (even if the edges have any number of boundary points) that are not compact.

There are three interesting examples related to Theorem 3. Two are based on the Knaster-Kuratowski example of a subset $K$ of the plane $\mathbb{R}^{2}$ which is connected, but for which there is a point $p$ such that $K \backslash\{p\}$ is totally disconnected. Specifically, let $C$ denote the Cantor set situated on $[0,1] \times\{0\}$ in $\mathbb{R}^{2}$; thus, $(x, 0) \in C$ if $x$ has a (possibly infinite) ternary representation that does not use a 1. Let $D \subseteq C$ consist of those points having a finite ternary expansion (possibly using a 1$)$, let $F=C \backslash D$ and let $p$ be the point $\left(\frac{1}{2}, 1\right)$. For each $c \in \mathbb{R} \times\{0\}$, let $S_{c}$ denote the straight segment joining $p$ and $c$. The Knaster-Kuratowski space 
$\mathbf{K}$ is the union of the sets

$$
L_{c}=\left\{\begin{array}{l}
\left\{(x, y) \in S_{c} \mid y \in \mathbb{Q}\right\}, c \in D \\
\left\{(x, y) \in S_{c} \mid y \notin \mathbb{Q}\right\}, c \in F .
\end{array}\right.
$$

The following facts are relevant [17]: $D$ is dense in $C ; \mathbf{K}$ is connected; $\mathbf{K} \backslash\{p\}$ has only trivial components; and the quasi-components of $\mathbf{K} \backslash\{p\}$ are the sets $L_{c}, c \in C$.

In the first version, Example 4.2.9 in [19], we start with $Y=\mathbf{K} \backslash\{p\}$ and let $G$ be a graph with vertex set $Y \cup\{\infty\}$, where $\infty$ is any point not in $Y$. The only edges we allow in $G$ will have $\infty$ as one end. Let $N(G)$ denote the neighbours of $\infty$, and let $Z(G)$ denote the $c \in C$ such that $N(G) \cap L_{c} \neq \emptyset$. Proposition 4.2.8 in [19] shows that $G$ is connected if and only if $Z(G)$ is dense in $C$.

Our specific example is obtained as follows. Let $d_{1}, d_{2}, \ldots$ be an enumeration of $D$. For each $i$, let $v_{i}$ be that point $(x, y)$ of $L_{d_{i}}$ such that $y=1-\frac{1}{2^{i}}$. Taking $v_{1}, v_{2}, \ldots$ for the neighbours of $\infty, G$ is a connected weakly Hausdorff space (actually "weakly regular") and yet has no minimal connected spanning set. This shows (not surprisingly) that some additional hypothesis, such as compactness or weak normality, is required.

The second example is $\mathbf{K}$ itself. No two points of $\mathbf{K}$ are contained in a minimal closed connected subset of $\mathbf{K}$, yet the edge space is weakly normal. In the context of trying to find fundamental cycles in a superspace having additional edges, such pairs present difficulties.

The third example is an edge space $(X, E)$ for which $X \backslash E$ consists of three closed points $u, v, w$. There are denumerably many edges in $E$, all having $\{u, v\}$ for boundary. The basic open sets containing $w$ consist of $w$ and all but finitely many edges. (They do not contain either $u$ or $v$.) Such a space is connected and compact, but has no minimal connected spanning set. It is not weakly Hausdorff - any neighbourhood of $u$ will contain each of the infinitely many edges, so $u, w$ cannot be finitely separated by open sets.

We shall need the following fact throughout this work.

Lemma 6. Let $H$ be a compact weakly Hausdorff space. Then, for any two components $K$ and $L$ of $H$, there is a separation $(A, B)$ of $H$ such that $K \subseteq A$ and $L \subseteq B$.

Kuratowski [14] (Section 47, Theorems 1 and 2) proves this for compact Hausdorff spaces. His proof applies in our context, but with some small modifications. One relevant point for the modification to weakly Hausdorff spaces is that, if $K$ is a component of $H$, then the intersection of all the open sets in $H$ containing $K$ is just $K$. Details are given in [19], Lemma 4.3.2 and Theorem 4.3.3.

\section{Cycle Spaces}

In this section, we introduce cycles in an edge space, the cycle and bond spaces of an edge space and prove that, for every compact weakly Hausdorff edge space: 
(1) every element of the cycle space is the edge-disjoint union of cycles; and (2) the space orthogonal to the bond space is the cycle space. The precise meanings of the ingredients of these theorems will be made clear through the rest of this section.

A cycle is a connected edge space $(X, E)$ such that $E \neq \emptyset$ and, for every $e \in E$, $X \backslash e$ is connected, but, for every distinct $e, f \in E, X \backslash\{e, f\}$ is not connected.

This surprisingly simple definition is clearly a characterization of classical cycles in graphs. It gives a very combinatorial flavour to the meaning of cycle, and so at least provides some kind of answer to the problem posed by Diestel and Kühn to find a combinatorial meaning for cycles.

The following lemmas may reassure the reader that our cycles have properties comparable to cycles in graphs. Corollary 9 perhaps does so even more. As we do not need the corollary in this work, its proof is omitted.

Lemma 7. Let $(X, E)$ be a cycle and let $F \subseteq E$ be finite and non-empty. Then $X \backslash F$ has $|F|$ components and there is a cyclic order $\left(K_{1}, \cdots, K_{|F|}\right)$ on these components so that, for each $i=1,2, \ldots,|F|$, there is an edge of $F$ with an incident vertex in each of $K_{i}$ and $K_{i+1}$ (indices taken modulo $|F|$ ).

The proof requires the following, expected, fact. As it is basic to the theory, we provide its proof.

Lemma 8. Let $(X, E)$ be an edge space, let $e \in E$ and let $K$ be the component of $X$ containing e. Then $K \backslash e$ has at most two components. If $K \backslash e$ has precisely two components, then each contains a vertex incident with e.

Proof. Suppose $(A, \bar{A})$ is a separation of $K \backslash e$ and that $A$ contains no vertex incident with $e$. Then $(A, \bar{A} \cup\{e\})$ is a separation of $K$, a contradiction. If $A$ has a separation $\left(A^{\prime}, A^{\prime \prime}\right)$, then $A^{\prime}$, say, cannot have a vertex incident with $e$ and $\left(A^{\prime}, A^{\prime \prime} \cup \bar{A} \cup\{e\}\right)$ is a separation of $K$, a contradiction.

Proof of Lemma \%. We proceed by induction on $|F|$. The case $|F|=1$ is trivial; we need separately the case $|F|=2$.

In this case, let $e, f \in F$. The definition of cycle and Lemma 8 tell us that $X \backslash e$ is connected, $X \backslash\{e, f\}$ has precisely two components and each of $e$ and $f$ has an incident vertex in each of the components.

If $|F| \geq 3$, delete an edge $e$ from $F$ to get $F^{\prime}$. Then $X \backslash F^{\prime}$ has $m=\left|F^{\prime}\right|$ components and a cyclic order $\left(K_{1}, \ldots, K_{m}\right)$ of them. For $i=1,2, \ldots, m$, there is an $e_{i} \in F^{\prime}$ having incident vertices in both $K_{i}$ and $K_{i+1}$. We may assume $e \in K_{m}$.

The definition tells us that $X \backslash\left\{e, e_{m}\right\}$ is disconnected; it has precisely two components $L$ and $M$. Let $L^{\prime}$ be the component of $K_{m} \backslash e$ containing a vertex incident with $e_{m-1}$. Since $Y=\left(K_{1} \cup \cdots \cup K_{m-1}\right) \cup L^{\prime} \cup\left\{e_{1}, \cdots, e_{m-1}\right\}$ is a connected subset of $X \backslash\left\{e, e_{m}\right\}$, it must be contained in $L$, say. Thus, $M \subseteq K_{m}$.

It follows that $K_{m} \backslash e$ has components $L^{\prime}$ and $M$. We know that $e_{m}$ has an incident vertex in each of $M$ and $K_{1}$. It follows that $\left(K_{1}, \cdots, K_{m-1}, L^{\prime}, M\right)$ is a 
cyclic sequence of the $|F|$ components of $X \backslash F$, such that consecutive components are joined by an edge of $F$.

Corollary 9. Let $(X, E)$ be a connected edge space with $|E| \geq 4$. The following are equivalent:

(1) $(X, E)$ is a cycle; and

(2) there is a cyclic order on $E$ such that, if $(w, x, y, z)$ is a cyclic subsequence, then $x$ and $z$ separate $w$ and $y$.

Let $(X, E)$ be an edge space. An edge cycle in $(X, E)$ is a subset $F$ of $E$ for which there is an edge subspace $(Y, F)$ of $(X, E)$ that is a cycle. (Recall this implies $F=Y \cap E$, and, for every $e \in F, \mathrm{Cl}_{X}(e) \subseteq Y$.)

Lemma 10. Let $(X, E)$ be a connected, compact, weakly Hausdorff edge space. Then, for every edge $e \in E$, either $X \backslash e$ is disconnected or else there exists an edge cycle of $X$ containing $e$, but not both.

Proof. Given any edge $e$, the subset $X \backslash e$ can only be disconnected if $e$ has two incident vertices, which are in distinct components after deleting $e$. Since an edge cycle containing $e$ arises from a cycle $C$ containing both vertices incident with $e$, and since $C \backslash e$ is a connected subset of $X \backslash e$, the two assertions cannot hold simultaneously.

If $e$ is incident with only one vertex, then $\mathrm{Cl}(e)$ is the required cycle. Otherwise, let $u, v$ be the vertices incident with $e$, and assume that $X \backslash e$ is connected. Then by Corollary 4 it contains a minimal closed connected edge subspace $P$ containing $\{u, v\}$. We claim that, if $f \in E \cap P$, then $u$ and $v$ are in different components of $P \backslash f$. Otherwise, let $K$ be the component of $P \backslash f$ containing $u$ and $v$. Since $f$ is open, $P \backslash f$ is closed in $X$, so $K$ is closed in $X$, contradicting minimality. It follows that $P \cup e$ is the required cycle.

We are now almost prepared to introduce the cycle space. A family $\mathcal{E}$ of subsets of a set $E$ is thin if each element of $E$ occurs in only finitely many elements of $\mathcal{E}$. The point is that if $\mathcal{E}$ is thin, then the symmetric difference $D$ of the sets in $\mathcal{E}$ is well-defined: an element $e$ of $E$ is in $D$ if and only if $e$ is in an odd number of elements of $\mathcal{E}$.

We have the following sets of subsets of $E$ generated by a family $\mathcal{E}$ of subsets of $E$ (thin or not):

(1) the weak span $\mathcal{W}(\mathcal{E})$ of $\mathcal{E}$ is the set of all symmetric differences of finitely many elements of $\mathcal{E}$

(2) the algebraic span $\mathcal{A}(\mathcal{E})$ of $\mathcal{E}$ is the set of all symmetric differences of thin subsets of $\mathcal{E}$; and

(3) the strong span $\mathcal{S}(\mathcal{E})$ is the smallest subset of the power set of $E$ that contains $\mathcal{E}$ and is closed under symmetric differences of thin families.

Obviously we have $\mathcal{W}(\mathcal{E}) \subseteq \mathcal{A}(\mathcal{E}) \subseteq \mathcal{S}(\mathcal{E})$. As a simple example, let $E=$ $\left\{e_{1}, e_{2}, \ldots\right\}$ be a countably infinite set and let $\mathcal{E}$ consist of the pairs $\left\{e_{1}, e_{i}\right\}$, 
$i=2,3, \ldots$ Then $\mathcal{W}(\mathcal{E})=\mathcal{A}(\mathcal{E})$ consists of all finite even subsets of $S$, while $\mathcal{S}(\mathcal{E})=\mathcal{A}(\mathcal{A}(\mathcal{E}))$ consists of all subsets of $S$, while (for any $\mathcal{E}) \mathcal{W}(\mathcal{W}(\mathcal{E}))=\mathcal{W}(\mathcal{E})$.

The cycle space $\mathcal{Z}=\mathcal{Z}(X, E)$ of an edge space $(X, E)$ is the strong span of the edge cycles of $(X, E)$. We shall see in Corollary 15 that, when $X$ is compact and weakly Hausdorff, the cycle space is also the algebraic span of the edge cycles of $(X, E)$; this is not obvious and is an important part of the statement of Theorem 14. (We note that Diestel and Kühn define their cycle space as the algebraic span of the edge sets of circles. Corollary 15 shows the two definitions are equivalent. Their version of this corollary is that the cycle space is closed under sums of thin families of elements of the cycle space.) The reader should also note that, for a finite graph with the classical topology, an edge cycle is the set of edges of a cycle and therefore the cycle space as defined here is the standard cycle space.

Notice that if $G$ is a graph (not necessarily locally finite), and $R$ and $S$ are rays in the same end of $G$ and have common origins, then the symmetric difference $R \triangle S$ of $R$ and $S$ is the symmetric difference of a thin family of finite cycles. Thus, for both definitions of the cycle space, $R \triangle S$ is in the cycle space. In fact, for any space $X$ containing $G, R \triangle S$ is in the cycle space of $X$. For more complicated elements of the cycle space of $\mathbf{F}(G)$, however, it is not clear whether they are elements of the cycle space of, say, the Stone-Cech compactification of $G$. Our theory applies equally well to the latter, but examples seem to be extremely difficult to analyze, since the Stone-Cech compactification is very poorly understood. We do not even know in detail what this compactification is for the natural numbers.

It seems that in order to study cycles, we must also study edge cuts. Let $A$ and $\bar{A}$ partition $X \backslash E$ into two closed sets. Then the set $\delta(A)$ of edges having one end in $A$ and one end in $\bar{A}$ is an edge cut. It is not obvious that, when $X$ is connected, edge cuts are cutsets; in fact, the third example in Section 2 (which is not weakly Hausdorff) provides a counterexample to this - namely the edge cut $\delta(w)$, which is empty. We shall show that edge cuts are cutsets in Section 5 (Corollary 25), at a level of generality which extends that of compact weakly Hausdorff edge spaces. This is why we insist that both $A$ and $\bar{A}$ be closed: we want to ensure that $X \backslash \delta(A)$ is not connected.

The following is a basic proposition that is not trivial in this context.

Lemma 11. Let $(X, E)$ be a compact, weakly Hausdorff edge space. Let $Z$ be an edge cycle of $(X, E)$ and let $B$ be an edge cut of $(X, E)$. Then $|Z \cap B|$ is finite and even.

We need the following crucial fact.

Theorem 12. Every edge cut in a compact weakly Hausdorff edge space is finite.

Proof. Let $(X, E)$ be the given edge space and let $A$ and $\bar{A}$ partition $X \backslash E$ into two closed sets. Since $X$ is compact, Lemma 2 implies $X$ is weakly normal and, therefore, there are open sets $U_{A}$ and $U_{\bar{A}}$ in $X$, containing $A$ and $\bar{A}$, respectively, such that $U_{A} \cap U_{\bar{A}}$ is finite. But $\delta(A) \subseteq U_{A} \cap U_{\bar{A}}$, so $\delta(A)$ is finite. 
Proof of Lemma 11. By Theorem 12, $B$ is finite, so $Z \cap B$ is finite. Let $(Y, Z)$ be a cycle. By Lemma $7, Y \backslash(Z \cap B)$ has finitely many components, joined cyclically in the order $K_{1}, K_{2}, \ldots, K_{r}$ by the edges of $Z \cap B$. Let $(A, \bar{A})$ be a partition of $X \backslash E$ into closed sets such that $B=\delta(A)$.

Every edge of $Z \cap B$ has one incident vertex in $A$ and the other in $\bar{A}$. Applying Lemma 7 , the sets $K_{i} \backslash E$ are alternately contained in $A$ and contained in $\bar{A}$. Therefore $r$ is even, as required.

It is interesting to compare this with earlier studies of cycle spaces for infinite graphs. In those cases, a cycle $Z$ is necessarily finite, while the cuts $B$ may be infinite. Of course $|Z \cap B|$ is even. In our context, it is the cycles that may be infinite, while the cuts are finite and we also have $|Z \cap B|$ even.

The bond space $\mathcal{B}=\mathcal{B}(X, E)$ of an edge space $(X, E)$ is the set of all cuts. It is very easy to see that, given separations $\left(A_{1}, \bar{A}_{1}\right),\left(A_{2}, \bar{A}_{2}\right)$ of $X \backslash E$ (so $A_{i}, \bar{A}_{i}$ are complementary and both simultaneously open and closed in $X \backslash E$, for $i=1,2$ ), we have that $\delta\left(A_{1} \triangle A_{2}\right)=\delta\left(A_{1}\right) \triangle \delta\left(A_{2}\right)$. It follows that the bond space is a vector space over $\mathbb{Z}_{2}$.

Furthermore, Theorem 12 implies that $\mathcal{B}$ is the weak span of the inclusionwise minimal edge cuts, which, when $X$ is a finite graph, are often called bonds, whence the name "bond space" for $\mathcal{B}$.

For any set $\mathcal{A}$ of subsets of a set $S$, the set $\mathcal{A}^{\perp}$ is defined to be the set of subsets $C$ of $S$ such that, for every $C^{\prime} \in \mathcal{A},\left|C \cap C^{\prime}\right|$ is even. Throughout this article, whenever we consider $\mathcal{A}^{\perp}$ for a set $\mathcal{A}$ whose elements are sets of edges of an edge space, $S$ will tacitly be assumed to be the set of all edges (and nothing more).

Corollary 13. If $(X, E)$ is a compact weakly Hausdorff edge space, then $\mathcal{Z} \subseteq \mathcal{B}^{\perp}$ and $\mathcal{B} \subseteq \mathcal{Z}^{\perp}$.

Proof. The two claims are trivially equivalent. To prove the first, it suffices to show that every edge cycle is in $\mathcal{B}^{\perp}$ and that $\mathcal{B}^{\perp}$ is closed under sums of thin families. The former is the content of Lemma 11. For the latter, suppose $A \subseteq \mathcal{B}^{\perp}$ is a thin family and that $b \in \mathcal{B}$. The set $A$ is thin and, by Theorem $12, b$ is finite, so the set $A^{\prime}=\{a \in A \mid a \cap b \neq \emptyset\}$ is finite. Clearly $b \cap\left(\triangle A^{\prime}\right)=b \cap(\triangle A)$. Since $A^{\prime}$ is finite, $\left|b \cap\left(\triangle A^{\prime}\right)\right| \equiv \sum_{a \in A^{\prime}}|b \cap a|(\bmod 2)$. Since every $|b \cap a|$ is even, $|b \cap(\triangle A)|$ is even, as required.

We are now ready to prove our first main result.

Theorem 14. Let $(X, E)$ be a compact, weakly Hausdorff edge space. Then:

(1) $\mathcal{B}^{\perp}=\mathcal{Z}$; and

(2) every element of the cycle space is the disjoint union of edge cycles.

Proof. For both of these, it suffices to prove that every element of $\mathcal{B}^{\perp}$ is the disjoint union of edge cycles. From this (1) follows, since it obviously implies $\mathcal{B}^{\perp} \subseteq \mathcal{Z}$ and Corollary 13 is the reverse inclusion. Conclusion (2) is now immediate from the two preceding sentences. 
So let $F \in \mathcal{B}^{\perp}$ and let $\mathcal{P}$ denote the set of all sets of pairwise disjoint edge cycles contained in $F$. That is, an element of $\mathcal{P}$ is a set of pairwise disjoint edge cycles, each contained in $F$. The union of any chain of elements of $\mathcal{P}$ is trivially again an element of $\mathcal{P}$, so Zorn's Lemma implies $\mathcal{P}$ has a maximal element $P$.

Let $F^{\prime}=\cup_{C \in P} C$. Corollary 13 implies that $F^{\prime} \in \mathcal{B}^{\perp}$. Hence $F^{\prime} \triangle F=F \backslash F^{\prime} \in$ $\mathcal{B}^{\perp}$.

Now suppose, by way of contradiction, that $F^{\prime} \triangle F$ has an edge $e$, and let $X^{\prime}=(X \backslash E) \cup\left(F^{\prime} \triangle F\right)$. Let $K$ be the component of $X^{\prime}$ containing $e$. If $K \backslash e$ is connected, then by Lemma $10 e$ is in an edge cycle $C$ of $X^{\prime}$, which is necessarily contained in $F^{\prime} \triangle F$. Since $F^{\prime} \triangle F=F \backslash F^{\prime}, P \cup\{C\}$ is a set of pairwise disjoint edge cycles, all contained in $F$, contradicting the maximality of $P$.

Thus, we may assume that $K \backslash e$ is not connected. We claim that $\{e\}$ is an edge cut of $X^{\prime}$. To see this, let $W=X^{\prime} \backslash e$. Then by Lemma $8 K$ has turned into two components $K_{1}$ and $K_{2}$ of $W$. From Lemma 6 there is a separation $(A, \bar{A})$ of $W$ such that $K_{1} \subseteq A$ and $K_{2} \subseteq \bar{A}$. Since $W=X^{\prime} \backslash e,\{e\}$ is an edge cut of $X^{\prime}$, as required.

Since $W$ contains all of $X \backslash E,(A, \bar{A})$ gives an edge cut of $X$, which is finite by Theorem 12 . Only finitely many edge cycles in $\mathcal{P}$ can meet $\delta(A)$, and each one does so in an even number of edges. Thus, $F^{\prime} \cap \delta(A)$ is even. On the other hand, $F$ has only one more edge in $\delta(A)$, namely $e$, so $F \cap \delta(A)$ is odd, contradicting the assumption that $F$ is orthogonal to every cut.

Corollary 15. Let $(X, E)$ be a connected, compact, weakly Hausdorff edge space. Then the cycle space is the algebraic span of the edge cycles.

\section{Fundamental Cycles}

In this section, we shall show that the fundamental cycles of a connected, compact, weakly Hausdorff edge space $(X, E)$ algebraically generate the cycle space. Let $T$ be a minimal connected spanning set for $X$, as guaranteed by Corollary 5, let $E^{\prime}=E \cap T$, and let $e^{*} \in E \backslash E^{\prime}$ have incident vertices $u$ and $v$. Then $u, v \in T$ and $T \cup e^{*}$ is a closed subset of $X$, whence $Y:=\left(T \cup e^{*}, E^{\prime} \cup e^{*}\right)$ is another connected, compact, weakly Hausdorff edge space. Since $T$ is connected, Lemma 10 implies that there exists an edge cycle $F \subseteq E$ of $Y$ (and therefore of $(X, E))$ containing $e^{*}$.

An easy argument similar to the one in the proof of Lemma 10 shows that, for any edge $e \in T \cap F$, the subset $T \backslash e$ is disconnected and $u$ and $v$ are in different components of $T \backslash e$. Therefore, the set $F \backslash e^{*}$ is precisely the set of edges of $T$ that separate $u$ and $v$ (in $T$ ). Hence the edge cycle $F$ is uniquely determined by $e^{*}{ }^{2}$ We refer to $F$ as the fundamental cycle of $e^{*}$ with respect to $T$, and denote it by $C_{e^{*}}$.

Our next goal is to show that the set of fundamental cycles is thin. This is done by considering the fundamental bonds: for each edge $e \subseteq T, T \backslash e$ has

\footnotetext{
${ }^{2}$ Although the same cannot be said of the cycle that determines $F$, i.e., the subset $C$ such that $(C, F)$ is a cycle and an edge-subspace of $Y$.
} 
precisely two components, which induce an edge cut $B_{e}$ containing $e$. (In fact this edge cut is minimal, i.e., is a bond.) By Theorem 12, $\left|B_{e}\right|$ is finite.

The following generalizes classical results and has a very simple proof, needing only that, if $e \in T \cap E$, then $T \backslash e$ has two components.

Lemma 16. Let $(X, E)$ be a connected, compact, weakly Hausdorff edge space. Let $T$ be a minimal connected spanning set for $(X, E)$, e an edge in $T$ and $f$ an edge not in $T$. Then $e \in C_{f}$ if and only if $f \in B_{e}$.

Theorem 17. Let $(X, E)$ be a connected, compact, weakly Hausdorff edge space. For any minimal connected spanning set $T$, the set of fundamental cycles is thin.

Proof. If the edge $e$ is not in $T$, then $e$ is only in $C_{e}$. If $e \in T$ is in $C_{f}$, then $f \in B_{e}$ and, since $\left|B_{e}\right|$ is finite, there are only finitely many such $C_{f}$ containing $e$, as required.

And now we have one of our main theorems.

Theorem 18. Let $(X, E)$ be a connected, compact, weakly Hausdorff edge space. Let $T$ be any minimal connected spanning set. If $z \in \mathcal{Z}(X, E)$, then

$$
z=\triangle_{e \in z \backslash T} C_{e} .
$$

That is, the fundamental cycles algebraically generate the cycle space.

Proof. By Theorem 17, the set $z^{\prime}=\triangle_{e \in z \backslash T} C_{e}$ is defined and is in the cycle space. Since the cycle space is closed under symmetric difference, $z \Delta z^{\prime}$ is in the cycle space. By definition of $z^{\prime}$, if $e$ is not in $T$, then $e \notin z \triangle z^{\prime}$, so $z \triangle z^{\prime} \subseteq T$. By Theorem 14 (2), if $z \triangle z^{\prime} \neq \emptyset$, there is an edge cycle $C \subseteq z \triangle z^{\prime}$. Thus, there is a cycle $(Y, C)$ in $(X, E)$. But $Y \subseteq T$ and deleting any edge of $T$ disconnects $T$, a contradiction.

We conclude this section with the following result, for which no analogue appears in the Diestel-Kühn works. A connected edge space $(X, E)$ is 2-edgeconnected if, for every $e \in E, X \backslash e$ is connected.

Theorem 19. Let $(X, E)$ be a 2-edge-connected, compact, weakly Hausdorff edge space. Then $\mathcal{Z}^{\perp}=\mathcal{B}$.

Proof. We have from Corollary 13 that $\mathcal{B} \subseteq \mathcal{Z}^{\perp}$. Let $T$ be a minimal connected spanning set for $(X, E)$ and let $\mathcal{F}$ be the set of fundamental cycles with respect to $T$.

Let $A \in \mathcal{Z}^{\perp}$. We claim that $A$ is finite. By way of contradiction, suppose not. Let $G$ be the simple bipartite graph with bipartition $(A, \mathcal{F})$ and an edge between $a \in A$ and $F \in \mathcal{F}$ if $a$ is an edge of $F$.

Notice that every $F \in \mathcal{F}$ has finite even degree in $G$, since $A \in \mathcal{Z}^{\perp}$. Since $\mathcal{F}$ is a thin family, for every $e \in E$, and so, in particular, for every $e \in A, e$ is in finitely many members of $\mathcal{F}$. Thus, every vertex in $A$ has finite degree in $G$.

Since $(X, E)$ is 2-edge-connected, by Lemma 10 every $e \in E$ is in some edge cycle and, therefore, in some element of $\mathcal{F}$. By assumption, $A$ is infinite, so the 
preceding sentence and paragraph imply $\mathcal{F}$ is infinite. It follows easily that there is an infinite subset $\mathcal{F}^{\prime}$ of $\mathcal{F}$ such that, if $F$ and $F^{\prime}$ are distinct elements of $\mathcal{F}^{\prime}$, $F \cap A$ and $F^{\prime} \cap A$ are disjoint and not empty.

Since $\mathcal{F}$ is thin, so is $\mathcal{F}^{\prime}$. Thus, the set $z=\triangle_{F \in \mathcal{F}^{\prime}} F$ is in $\mathcal{Z}$. The definition implies $A \cap z$ is even; in particular it is finite. But $\mathcal{F}^{\prime}$ was selected so that no two elements of $\mathcal{F}^{\prime}$ have any elements of $A$ in common. So $z$ contains all the elements of $A$ contained in some member of $\mathcal{F}^{\prime}$. Since every element of $\mathcal{F}^{\prime}$ has an element of $A$ and there are infinitely many elements of $\mathcal{F}^{\prime}, z$ has infinitely many elements of $A$, a contradiction.

So $A$ is finite. Let $H$ be the graph whose vertices are the finitely many components of $X \backslash A$ and whose edges are the edges in $A$, having as ends the components containing their ends in $X$. It is easy to use Lemma 7 and Theorem 3 to transform graphical cycles (i.e., classical cycles in finite graphs) in $H$ to edge cycles in $(X, E)$. If the cycle in $H$ has $k$ edges, then the corresponding edge cycle $D$ in $(X, E)$ has $|D \cap A|=k$. Since $A \in \mathcal{Z}^{\perp}$, we conclude that $k$ must be even. Thus, $H$ has only even cycles, i.e., $H$ is bipartite.

Let $K_{1}$ be the union of components of $X \backslash A$ on one side of the bipartition of the vertices of $H$ and let $K_{2}$ be the union of the remaining components of $X \backslash A$. Then $\left(K_{1} \backslash E, K_{2} \backslash E\right)$ is a partition of $X \backslash E$ into two closed sets, and $A=\delta\left(K_{1} \backslash E\right)$, as required.

A simple, relevant example is the Freudenthal compactification of a ray, which consists of a ray plus one additional point. This space is connected, compact and weakly Hausdorff. However, $\mathcal{Z}=\{\emptyset\}, \mathcal{B}$ is the set of finite sets of edges, while $\mathcal{Z}^{\perp}$ is the set of all subsets of edges. The following corollary shows that having infinitely many cut edges is the only time that $\mathcal{Z}^{\perp}$ is not $\mathcal{B}$.

Corollary 20. Let $(X, E)$ be a connected, compact, weakly Hausdorff edge space. Let $E^{\prime}=\{e \in E \mid X \backslash e$ is disconnected $\}$. Then $\mathcal{Z}^{\perp}=\mathcal{B}\left(X, E \backslash E^{\prime}\right) \oplus 2^{E^{\prime}}$.

Here, the $\oplus$ means that every set in $\mathcal{Z}^{\perp}$ is the disjoint union of a set in $\mathcal{B}\left(X, E \backslash E^{\prime}\right)$ and a set in $2^{E^{\prime}}$, which is the set of all subsets of $E^{\prime}$. The proof is interesting in that it uses the freedom we have to declare which edges we consider. In this case, it is the cut-edges we ignore. The reader should realize that this is contraction of edges. A feature of our model, however, is that we do not have to change the space, just the set of "recognized" edges.

Proof. If $Z \in \mathcal{Z}$, then $Z \cap E^{\prime}=\emptyset$, so every subset of $E^{\prime}$ is in $\mathcal{Z}^{\perp}$. More generally, $\mathcal{Z}^{\perp}$ is of the form $\mathcal{X} \oplus 2^{E^{\prime}}$, where every element of $\mathcal{X}$ is contained in $E \backslash E^{\prime}$.

Furthermore, $\mathcal{Z}(X, E)=\mathcal{Z}\left(X, E \backslash E^{\prime}\right)$. Since $\left(X, E \backslash E^{\prime}\right)$ is 2-edge-connected, Theorem 19 implies $\left[\mathcal{Z}\left(X, E \backslash E^{\prime}\right)\right]^{\perp}=\mathcal{B}\left(X, E \backslash E^{\prime}\right)$. Putting these two paragraphs together, the result follows. 


\section{More GENERAL EDGES}

In studying embeddings of graphs in surfaces, the graph is identified with a subspace of the surface in which each edge corresponds to an open arc having two points in its boundary. This is, of course, the 1-dimensional cell complex associated with the graph. In the literature on continuum theory, a closed subset $V$ of a continuum $X$ is a $T$-set if the components of $X \backslash V$ are open sets having precisely two boundary points. If $V$ is a $T$-set in a continuum $X$, then $(X, X \backslash V)$ has a structure quite analogous to an edge space.

In this section, we provide a general quotient operation that turns a compact, weakly Hausdorff space with connected open sets as "edges", such as $(X, X \backslash V)$ in the preceding paragraph, into a compact, weakly Hausdorff edge space. The main point is that the cycle spaces of the two spaces are isomorphic and the cuts are in 1-1 correspondence. Thus, the theory developed in Sections 3 and 4 extends to include such spaces. This will not be quite enough to show that the Diestel-Kühn cycle space is the same as ours - we will also have to show that their circles correspond to our cycles.

Our quotient operation will not only produce an edge space $(X, E)$, which means the elements of $E$ are open singletons, but the components of $X \backslash E$ will also be singletons. We will see an example in Section 6 in which this point is instructive.

There is a second example of this type. The binary tree has a compactification in which the ends form an interval (there is a continuous function from the Cantor set to the unit interval). The quotient operation applied to this compactification is the Alexandroff compactification of the binary tree.

A generalized edge space is a pair $(X, \mathcal{E})$ consisting of a topological space $X$ together with a set $\mathcal{E}$ of pairwise disjoint, connected, open, but not closed, subsets of $X$, each having at most two additional points in its closure. We denote the subspace $X \backslash\left(\cup_{e \in \mathcal{E}} e\right)$ by $X-\mathcal{E}$. The concepts of edges, edge cycles, cycles, cycle spaces, fundamental cycles, cuts, bonds and bond spaces extend in the obvious way to their "generalized" version.

Let $(X, \mathcal{E})$ be a generalized edge space. The edge-vertex decomposition $\mathcal{D}$ of $X$ is the partition of $X$ into the connected open sets in $\mathcal{E}$ and the connected closed sets that are the components of $X-\mathcal{E}$. The simplification of $(X, \mathcal{E})$ is the pair $(Y, F)$, where $Y$ is the topological quotient $X / \mathcal{D}$ whose points are the sets in $\mathcal{D}$, and $F \subseteq Y$ being precisely the parts from $\mathcal{E}$.

The crucial relationships are given in the following theorem, the first main point of this section. Recall that, for a partition $\mathcal{P}$ of a topological space $X$ :

(1) a set $Y \subseteq X$ is saturated if $Y$ is the union of sets in $\mathcal{P}$; and

(2) $\mathcal{P}$ is upper semicontinuous if, for every part $P$ and every open set $U$ of $X$ for which $P \subseteq U$, then there is a saturated open set $V$ such that $P \subseteq V \subseteq U$.

Theorem 21. Let $(X, \mathcal{E})$ be a compact, weakly Hausdorff generalized edge space. Then the decomposition of $X-\mathcal{E}$ into components is upper semicontinuous. If 
$(Y, F)$ is the simplification of $(X, \mathcal{E})$ and $p: X \rightarrow Y$ is the associated quotient map, then:

(1) $(Y, F)$ is a compact, weakly Hausdorff edge space; and

(2) if $A \subseteq Y$, then $A$ is connected in $Y$ if and only if $p^{-1}(A)$ is connected in $X$.

In general, it is not true that the edge-vertex decomposition of an edge space $(X, \mathcal{E})$ is upper semicontinuous. When $X$ is a compact metric space, that the decomposition of $X-\mathcal{E}$ is upper semicontinuous is [4, Th. 8.F.14]. The proof is readily adapted to compact weakly Hausdorff spaces [19, Thm. 4.3.16].

Intermediate between the generalized edge space $(X, \mathcal{E})$ and its simplification $(Y, F)$ is the edge simplification $\left(Y^{\prime}, F^{\prime}\right)$, in which each element of $\mathcal{E}$ is reduced to a single point. Thus, the components of $Y^{\prime} \backslash F^{\prime}$ and $X \backslash \mathcal{E}$ are the same, and the edges in $F$ and $F^{\prime}$ can be interpreted as being the same. The two statements in Theorem 21 concerning $(Y, F)$ also apply (rather more easily) to $\left(Y^{\prime}, F^{\prime}\right)$.

In order to prove the rest of Theorem 21, we need some preliminaries.

Lemma 22. Let $(X, \mathcal{E})$ be a compact generalized edge space and $\left(A_{1}, A_{2}\right)$ a separation of $X-\mathcal{E}$. For $i=1,2$, let $U_{i}$ be an open set containing $A_{i}$ and disjoint from $A_{3-i}$. Let $\mathcal{M}$ and $\mathcal{N}$ denote the sets of generalized edges $\left\{e \in \mathcal{E} \mid e \backslash\left(U_{1} \cup U_{2}\right) \neq \emptyset\right\}$ and $\left\{e \in \mathcal{E} \mid \mathrm{Cl}(e) \cap U_{1} \neq \emptyset\right.$ and $\left.\mathrm{Cl}(e) \cap U_{2} \neq \emptyset\right\}$ respectively. Then $\mathcal{M}$ is finite, and if $U_{1} \cap U_{2}$ is finite, then so is $\mathcal{N}$.

Proof: Suppose first that the set $\mathcal{M}$ is infinite, and for every $e \in \mathcal{M}$ choose $x_{e} \in e \backslash\left(U_{1} \cup U_{2}\right)$. Since $X$ is compact, the set $\left\{x_{e}\right\}_{e \in \mathcal{M}}$ has an accumulation point $x$. Since the (generalized) edges are open and pairwise disjoint, $x$ is not in any edge, that is, $x \in X-\mathcal{E}$. Thus, for some $i \in\{1,2\} x \in A_{i}$. Then $U_{i}$ is an open set containing $x$, but no $x_{e}$ is in $U_{i}$, contradicting the fact that $x$ is an accumulation point for $\left\{x_{e}\right\}_{e \in \mathcal{E}}$.

Hence $\mathcal{M}$ is finite. Suppose now that $U_{1} \cap U_{2}$ is finite. It is sufficient to show that $\mathcal{L}:=\mathcal{N} \backslash \mathcal{M}$ is finite. For any edge $e$ and $i=1,2$, since $U_{i}$ is open, $\mathrm{Cl}(e) \cap U_{i} \neq \emptyset$ implies that $e \cap U_{i} \neq \emptyset$. So for $e \in \mathcal{L}$ we have that $e \subseteq\left(U_{1} \cup U_{2}\right)$, $e \cap U_{1} \neq \emptyset \neq e \cap U_{2}$. Since $e$ is connected, $\left(U_{1} \cap e, U_{2} \cap e\right)$ is not a separation of $e$, that is, deleted $\left(e \cap U_{1} \cap U_{2}\right) \neq \emptyset$. Since $U_{1} \cap U_{2}$ is finite and the generalized edges are disjoint, $\mathcal{L}$ is finite.

In the next result, for a separation $\left(A_{1}, A_{2}\right)$ of $X-\mathcal{E}, \delta\left(A_{1}\right)$ denotes the generalized cut consisting of all the edges such that $\left(\mathrm{Cl}(e) \cap A_{1}\right) \neq \emptyset$ and $(\mathrm{Cl}(e) \cap$ $\left.A_{2}\right) \neq \emptyset$.

Corollary 23. Let $(X, \mathcal{E})$ be a compact weakly Hausdorff generalized edge space, and let $\left(A_{1}, A_{2}\right)$ be a separation of $X-\mathcal{E}$. Then $\delta\left(A_{1}\right)$ is finite.

Proof: Since $X$ is weakly normal, there are open sets $U_{1}$ and $U_{2}$ in $X$ containing $A_{1}$ and $A_{2}$, respectively, such that $U_{1} \cap U_{2}$ is finite. Furthermore, $A_{1}$ and $A_{2}$ are closed in $X$, so we may assume $U_{1} \cap A_{2}=\emptyset$ and $U_{2} \cap A_{1}=\emptyset$. Then the set $\mathcal{N}$, 
as defined in Lemma 22 is finite (by the same lemma). But $\delta\left(A_{1}\right) \subseteq \mathcal{N}$. Hence $\delta\left(A_{1}\right)$ is finite.

Our next lemma gives a relationship between the topology on $X-\mathcal{E}$ and $X$. For a set $A$ that is open and closed in $X-\mathcal{E}$, we set $A^{\square}$ to be the set $A \cup\{e \in \mathcal{E} \mid \operatorname{Cl}(e) \cap A \neq \emptyset\}$.

Lemma 24. Let $(X, \mathcal{E})$ be a compact, weakly Hausdorff generalized edge space and let $A \subseteq X-\mathcal{E}$ be both open and closed in $X-\mathcal{E}$. Then $A^{\square}$ is open in $X$.

In fact, we can describe the technical property that is required for Lemma 24. Let $(X, E)$ be an edge space and let $E^{\prime} \subseteq E$. A set $W \subseteq X$ is a transversal of $E^{\prime}$ if every edge in $E^{\prime}$ has an end in $W$. The edge space $(X, E)$ is quasi-regular if, for any $E^{\prime} \subset E$ and any transversal $W$ of $E^{\prime}, \mathrm{Cl}\left(\bigcup_{e \in E^{\prime}} e\right) \backslash\left(\bigcup_{e \in E^{\prime}} \mathrm{Cl}(e)\right) \subseteq \mathrm{Cl}(W)$. Theorem 3.4.3 in [19] shows that Lemma 24 holds if $(X, E)$ is a quasi-regular edge space. It is also true that any weakly normal edge space is quasi-regular. We do not prove either of these facts here.

Proof of Lemma 24: Let $A^{\prime}$ be the complement of $A$ in $X-\mathcal{E}$. As in the proof of Corollary 23, there exist open sets $U, U^{\prime}$ containing $A, A^{\prime}$ and disjoint from $A^{\prime}, A$, respectively. We partition $\mathcal{E}$ into the three (possibly empty) sets $\mathcal{F}_{1}, \mathcal{F}_{2}$ and $\mathcal{F}_{3}$, where $\mathcal{F}_{1}$ is the set of edges $e \in \mathcal{E}$ such that $e \nsubseteq U \cup U^{\prime}$, and $\mathcal{F}_{2}$ the set of edges $e$ such that $e \subseteq U \cup U^{\prime}$ and $\mathrm{Cl}(e) \cap U \neq \emptyset \neq \mathrm{Cl}(e) \cap U^{\prime}$. By Lemma 22 $\mathcal{F}_{1}$ and $\mathcal{F}_{2}$ are finite.

Note that any edge of $\mathcal{F}_{3}$ is entirely contained in precisely one of $U, U^{\prime}$ (and is disjoint from the other), according to whether its ends are in $A$ or $A^{\prime}$ respectively. Thus, every such edge is in $A^{\square}$ if and only if it is contained in $U$. As for the edges in $\mathcal{F}_{1} \cup \mathcal{F}_{2}$, we observe that if such an edge $e$ is not (entirely) in $A^{\square}$, then $\mathrm{Cl}(e)$ is disjoint from $A^{\square}$. Hence

$$
A^{\square}=\left(U \cup \bigcup_{e \in \mathcal{F}_{1} \cup \mathcal{F}_{2}: e \subseteq A^{\square}} e\right) \backslash\left(\bigcup_{e \in \mathcal{F}_{1} \cup \mathcal{F}_{2}: e \nsubseteq A^{\square}} \mathrm{Cl}(e)\right) .
$$

Since $\mathcal{F}_{1} \cup \mathcal{F}_{2}$ is finite, $A^{\square}$ is open.

Here is the fact that cuts disconnect the space, promised preceding Lemma 11.

Corollary 25. Let $(X, \mathcal{E})$ be a compact weakly Hausdorff generalized edge space. If $\mathcal{F}$ is an edge cut, then $X-\mathcal{F}$ is disconnected.

Proof: If $\mathcal{F}=\delta\left(A_{1}\right)=\delta\left(A_{2}\right)$ for some separation $\left(A_{1}, A_{2}\right)$ of $X-\mathcal{E}$, then $\left(A_{1}^{\square}-\mathcal{F}, A_{2}^{\square}-\mathcal{F}\right)$ is a separation of $X-\mathcal{F}$.

The following gives the final ingredient for the proof of Theorem 21.

Lemma 26. Given $(X, \mathcal{E}),(Y, F)$ and $p: X \rightarrow Y$ as in Theorem 21, for every $f \in F, u \in Y \backslash F$ we have that $u \in \mathrm{Cl}(f)$ if and only if there exists some point $v \in X$ such that $p(v)=u$ and $v \in \mathrm{Cl}\left(p^{-1}(f)\right)$. 
Proof: One direction holds simply by virtue of the continuity of $p$. For the other direction, suppose that $u \in \mathrm{Cl}(f)$. Let $e \in \mathcal{E}$ be such that $p(e)=f$ and let $K$ be the component of $X-\mathcal{E}$ such that $p(K)=u$. It suffices to show that $\mathrm{Cl}(e) \cap K \neq \emptyset$.

Suppose not. Let $W$ be a component of $X-\mathcal{E}$ such that $\operatorname{Cl}(e) \cap W \neq \emptyset$. By Lemma 6 , there is a separation $\left(A_{W}, A_{W}^{\prime}\right)$ of $X-\mathcal{E}$ such that $K \subseteq A_{W}$ and $W \subseteq A_{W}^{\prime}$. There are at most two such $W$, so there is an open and closed (in $X-\mathcal{E})$ set $A$ containing $K$ and disjoint from every component containing an end of $e$.

Lemma 24 implies $A^{\square}$ is open in $X$. Since $A^{\square}$ is the union of parts of $\mathcal{D}, p\left(A^{\square}\right)$ is open in $Y$. But this is an open set in $Y$ containing $u=p(K)$ and disjoint from $f$, contradicting the assumption that $u \in \mathrm{Cl}(f)$.

Proof of Theorem 21: We have already seen that the decomposition of $X-\mathcal{E}$ into components is upper semicontinuous.

Let $\mathcal{D}$ be the edge-vertex decomposition of $X$. Since $p$ is continuous, $Y=X / \mathcal{D}$ is compact.

The points of $F$ are open in $Y$ and the points in $Y \backslash F$ are closed in $Y$. To show that $(Y, F)$ is an edge space, we must show that each $f \in F$ has one or two additional points in its closure; this follows immediately from Lemma 26 (note that no point in $F$ can be in the closure of any other point).

Next, we show $Y$ is weakly Hausdorff. Let $u_{1}, u_{2}$ be distinct points in $Y$. If $u_{i}$ is open in $Y$, then trivially $\left\{u_{i}\right\}$ and $Y$ are open sets, containing $u_{i}$ and $u_{3-i}$, respectively, such that $\left\{u_{i}\right\} \cap Y$ is finite. So we can assume both $u_{1}$ and $u_{2}$ are closed. For $i=1,2$, let $K_{i}$ be the component of $X-\mathcal{E}$ such that $p\left(K_{i}\right)=u_{i}$.

Since $K_{1}$ and $K_{2}$ are disjoint components of $X-\mathcal{E}$, Lemma 6 implies there is a separation $\left(A_{1}, A_{2}\right)$ of $X-\mathcal{E}$ such that, for $i=1,2, K_{i} \subseteq A_{i}$. Again, $p\left(A_{i}^{\square}\right)$ is open in $Y$ and $p\left(A_{1}^{\square}\right) \cap p\left(A_{2}^{\square}\right)$ consists of precisely those edges $f$ such that $p^{-1}(f)$ is a generalized edge in $\delta\left(A_{1}\right)$. By Corollary $23 \delta\left(A_{1}\right)$ is finite. Therefore, $Y$ is weakly Hausdorff.

Finally, if $p^{-1}(A)$ is connected, then the continuity of $p$ implies $A$ is connected. Conversely, suppose $A$ is connected and $(U, V)$ is a separation of $p^{-1}(A)$. Let $U^{\prime}, V^{\prime}$ be open in $X$ such that $U=U^{\prime} \cap p^{-1}(A)$ and $V=V^{\prime} \cap p^{-1}(A)$.

Since the decomposition of $X-\mathcal{E}$ into components is upper semicontinuous, for each component $K$ of $X-\mathcal{E}$, if $K \subseteq U^{\prime}$, then there is an open set $U_{K} \subseteq U^{\prime}$ of $X$ containing $K$ and every component of $X-\mathcal{E}$ that $U_{K}$ meets. Let $U^{\prime \prime}$ be the union of the $U_{K}$, over all components $K$ contained in $U^{\prime}$. Similarly, obtain $V^{\prime \prime}$ from the components of $X-\mathcal{E}$ contained in $V^{\prime}$.

If $e$ is an edge of $A$, then, since $p^{-1}(e)$ is connected, it is contained in one of $U$ and $V$. Let $U^{*}$ be the set obtained from $U^{\prime \prime}$ by adding all the edges of $X$ which have non-empty intersection with $U^{\prime \prime}$, and similarly obtain $V^{*}$ from $V^{\prime \prime}$. Then $U^{*}$ and $V^{*}$ are open (being the union of open sets) and $U^{*} \cap p^{-1}(A)$ and $V^{*} \cap p^{-1}(A)$ are disjoint. Moreover, for any edge $e \in A$, unless $A=\{e\}$, since $A$ is connected $A$ must contain an end of $e$, whence $p^{-1}(A)$ contains an end of 
$p^{-1}(e)$, which is contained in one of $U, V$, and therefore one of $U^{\prime \prime}$ or $V^{\prime \prime}$. Hence $p^{-1}(e) \subseteq U^{*} \cup V^{*}$. So $U^{*}, V^{*}$ together cover $p^{-1}(A), p\left(U^{*}\right)$ and $p\left(V^{*}\right)$ are open in $Y$ and $\left(p\left(U^{*}\right) \cap A, p\left(V^{*}\right) \cap A\right)$ is a separation of $A$, a contradiction.

Notice that, if $(X, \mathcal{E})$ is a compact, weakly Hausdorff generalized edge space, with simplification $(Y, F)$, then Theorem 21 implies that, for every cycle $\left(C, F^{\prime}\right)$ in $(Y, F),\left(p^{-1}(C), p^{-1}\left(F^{\prime}\right)\right)$ is a cycle in $(X, \mathcal{E})$. However, it is not generally true that if $\left(C, \mathcal{E}^{\prime}\right)$ is a cycle in $(X, \mathcal{E})$, then $\left(p(C), p\left(\mathcal{E}^{\prime}\right)\right)$ is a cycle in $(Y, \mathcal{F})$. Consider, for example, an edge space $(X, \mathcal{E})$ consisting of two edges and one large component (say a disc) of $X-\mathcal{E}$. We can get a cycle using both edges and arcs in the disc joining appropriately the ends of the edges, but the quotient of such a cycle is not a cycle in the simplification.

The other main point of this section is the following. Of course an analogous theorem holds for the edge simplification.

Theorem 27. Let $(X, \mathcal{E})$ be a compact, weakly Hausdorff generalized edge space and let $(Y, F)$ be its simplification, with quotient map $p: X \rightarrow Y$. Let $\hat{p}: 2^{\mathcal{E}} \rightarrow 2^{F}$ be defined by $\hat{p}\left(E^{\prime}\right)=\left\{p(e) \mid e \in E^{\prime}\right\}$. Then $\hat{p}: \mathcal{Z}(X, \mathcal{E}) \rightarrow \mathcal{Z}(Y, F)$ and $\hat{p}: \mathcal{B}(X, \mathcal{E}) \rightarrow \mathcal{B}(Y, F)$ are isomorphisms.

Proof: Part (2) of Theorem 21 and Corollary 25 together imply that $\hat{p}$ maps the edge cuts of $(X, \mathcal{E})$ bijectively onto those of $(Y, F)$, and, as observed above, if $C \subseteq F$ is an edge cycle in $(Y, F)$, then $\hat{p}^{-1}(C)$ is an edge cycle in $(X, \mathcal{E})$. We complete the proof by showing that, if $\mathcal{D} \subseteq \mathcal{E}$ is an edge cycle in $(X, \mathcal{E})$, then $\hat{p}(\mathcal{D})$ is in the cycle space of $(Y, F)$. By part $(1)$ of Theorem $21,(Y, F)$ satisfies the assumptions of Theorem 14. It is therefore sufficient to show that, for every edge cut $A=\delta(M)$ of $(Y, F), \hat{p}(\mathcal{D}) \cap A$ is finite and even.

By Lemma $26 \hat{p}^{-1}(A)$ is the edge cut $\delta\left(p^{-1}(M)\right)$. By Corollary $23 \mathcal{D} \cap \hat{p}^{-1}(A)$ is finite. We now observe that Lemma 7 (as well as Lemma 8, upon which it depends) holds for generalized edges - the proofs carry over simply by inserting "generalized" in front of "edges" throughout. It now follows, just as in the proof of Lemma 11, that $\mathcal{D} \cap \hat{p}^{-1}(A)$ is even. We conclude that $\hat{p}(\mathcal{D}) \cap A$ is finite and even.

\section{Remarks about the Diestel-KüHn SPACE $\widetilde{G}$}

In this section, we show that the most general graphs considered by Diestel and Kühn are actually examples of our theory, that is, the corresponding edge simplifications are compact and weakly Hausdorff and the two notions of cycle space coincide.

Let $G$ be a graph, let $v$ be a vertex of $G$ and let $\omega$ be an end of $G$. Then $v$ dominates $\omega$ if, for some (and hence every) ray $R$ in $\omega$, there are infinitely many $v R$-paths, disjoint except for their common end $v$. If $G$ has the property that

no end is dominated by more than one vertex, then $\widetilde{G}$ is the topological space obtained from $\mathbf{F}(G)$ by identifying each end with any vertex that dominates it. 
In their theory of cycle spaces, the most general kind of graph $G$ that Diestel and Kühn consider is one in which no two vertices are joined by infinitely many internally disjoint paths [10] and they develop their cycle space theory for the corresponding space $\widetilde{G}$. (One or two technical theorems along the way are proved for more general graphs, but their cycle space theory seems to require this assumption.)

A graph $G$ is finitely connected if, for every pair of vertices $u$ and $v$ of $G$, there is no set of infinitely many internally disjoint $u v$-paths in $G$.

The main results in this section are the following theorems, which show that our theory applies to the Diestel-Kühn spaces. Let $\bar{G}$ and $\widehat{G}$ denote the edge simplifications of $\mathbf{F}(G)$ and $\widetilde{G}$, respectively, obtained by replacing each edge with an open singleton.

Theorem 28. If $G$ is finitely connected and 2-connected, then $\widetilde{G}$ and $\widehat{G}$ are compact.

Theorem 29. If $G$ is finitely connected and 2-connected, then $\widetilde{G}$ is Hausdorff and $\widehat{G}$ is weakly Hausdorff.

Theorem 30. If $G$ is finitely connected and 2-connected, then the cycle spaces of $\widetilde{G}$ and $\widehat{G}$ are the same.

In the last of these, the assumption of 2-connectedness may be omitted by the expedient of considering each block of $G$ and noting that the cycle spaces of $\widetilde{G}$ and $\widehat{G}$ are direct sums of the cycle spaces arising from all the blocks. For $\widetilde{G}$, Theorem 29 is a special case of Theorem 4.7 in [10]; we shall give a much shorter proof here for finitely connected graphs.

We first consider compactness. The proof of compactness in [5] is easily adapted to a more general situation. Following Diestel and Kühn, we define a direction of a topological space $X$ to be a function $d$ which assigns, to every closed compact set $K$, a component of $X \backslash K$, in such a way that $K \subseteq K^{\prime}$ implies that $d(K) \supseteq d\left(K^{\prime}\right)$. (Diestel and Kühn consider only Hausdorff spaces, in which all compact sets are closed.)

Directions were introduced in [11] as a topological concept which is defined for arbitrary Hausdorff spaces and which, for arbitrary graphs, corresponds precisely to that of a graph-theoretic end (in the sense of Halin), as opposed to Freudenthal's original (topological) concept of an end, which is only equivalent to that of an undominated end. Freudenthal's concept is defined in terms of decreasing sequences of open connected subsets with compact frontier. Under appropriate assumptions, for example if the space is locally connected, such sets can equivalently be thought of as components of complements of closed compact subsets, as pointed out by Diestel and Kühn (although the possibility of nonlocally-connected spaces, where this can fail, seems to have been overlooked).

We construct the direction extension $\mathrm{D}(X)$ of $X$ by emulating the construction of $\mathbf{F}(G)$, using directions instead of ends (this space was not considered in [11]). 
Instead of local connectedness, we assume now that the complement of any closed compact set consists of finitely many components; this ensures in particular that these components are open. Let $\Omega$ be the set of directions. We define a topology on $\mathbf{D}(X)=X \cup \Omega$ by keeping the same basic neighbourhoods of the points of $X$ and, for each point $d \in \Omega$, and for each closed compact set $K$ in $X$, we have the basic neighbourhood consisting of the component $d(K)$ and all directions $f$ such that $f(K)=d(K)$. Evidently, $X$ is a subspace and, when $X$ is a graph, $\mathbf{D}(X)=\mathbf{F}(X)$.

The reader should have no difficulty adapting [5] to prove the following.

Theorem 31. Let $X$ be a topological space such that the complement of any closed compact subset consists of finitely many components. Then $\mathbf{D}(X)$ is compact.

When $X$ is either a graph equipped with the classical topology, or the usual cell-complex associated with the graph, we may take the closed compact subsets to be simply finite subsets of vertices. Notice that the assumption that the complement has only finitely many topological components implies that each pair of points in $X \backslash E$ has at most finitely many edges joining them.

Also, for graphs with the classical or the cell-complex topology, the fact that deleting finitely many vertices leaves finitely many components is a necessary condition for $\mathbf{F}(G)$ to be compact - if, for some finite set $W$ of vertices, $X \backslash W$ has infinitely many components, then each component of $X \backslash W$ is open and this is most of an open cover of $X$ that has no finite subcover. (In the classical case, infinitely many components should contain a vertex. For example, the graph consisting of two vertices joined by infinitely many edges is compact in the classical topology.) In particular, for a graph $G, \mathbf{F}(G)$ is compact if and only if, for every finite set $W$ of vertices of $G, G-W$ has only finitely many components. The following shows then that if $G$ is finitely connected and 2connected, then $\widetilde{G}$ is compact.

Lemma 32. If $G$ is a finitely connected 2-connected graph, then $G$ is countable and, for every finite subset $W$ of $V(G), G-W$ has only finitely many components.

Proof: We first show that if $U$ is any infinite set of vertices in $G$, then there is a ray $R$ and infinitely many disjoint $U R$-paths. The relevance is the following easy fact, whose proof is omitted.

Lemma 33. For any graph $G$, the following are equivalent.

(1) For every finite subset $W$ of $V(G), G-W$ has finitely many components.

(2) For any infinite set $U$ of vertices in $G$, there is a ray $R$ in $G$ and infinitely many disjoint $U R$-paths.

By Lemma 8.2.2 in [6], the alternative is that there is a vertex $u$ with infinitely many $u U$-paths, disjoint except for $u$. Let $U^{\prime}$ be the infinite set of terminal vertices in $U$ of such a set of paths. But $G-u$ is connected, so the same lemma implies that either the desired ray exists (for $U^{\prime}$ and, therefore, for $U$ ) or there 
is a vertex $v$ and infinitely many $v U^{\prime}$-paths in $G-u$, disjoint except for $v$. If the latter occurs, then $u$ and $v$ are joined by infinitely many internally disjoint paths, contradicting the assumption that $G$ is finitely connected.

For countability, it suffices to show every vertex has countable degree, so suppose $u$ is a vertex of $G$ with uncountable degree. Because $G$ is 2-connected, $G-u$ is connected. There is a minimal tree $T$ in $G-u$ containing all the neighbours of $u$. Since $T$ is uncountable, it has a vertex $v$ of uncountable degree. By minimality, each component of $T-v$ must contain a neighbour of $u$, so $u$ and $v$ are joined by uncountably many internally disjoint paths, a contradiction.

An immediate consequence of Theorem 31 and Lemma 32 is the following.

Corollary 34. If $G$ is finitely connected and 2-connected, then $\mathbf{F}(G), \bar{G}, \widetilde{G}$, and $\widehat{G}$ are compact.

We now consider the Hausdorff properties.

Proof of Theorem 29. Let $E$ and $F$ be distinct parts in $\mathbf{F}(G)$. If $E$ is just a point in the interior of an edge, then $E$ has a basic neighbourhood whose closure is disjoint from $F$. So we consider distinct points $x, y \in V(G) \cup \Omega$, where $\Omega=\mathbf{F}(G) \backslash G$ is the set of ends of $G$. The crucial point that remains is that, if (and in fact only if) $x$ and $y$ are in different parts, then there is a finite set $S$ of vertices, plus a point from each edge joining $x$ and $y$, such that $x$ and $y$ are in different topological components of $\mathbf{F}(G) \backslash S$. It is then easy to see (replacing $x$ and $y$ with vertex-representatives of their equivalence classes, if necessary) that $S$ is disjoint from these two equivalence classes, so Lemma 39 provides the desired open sets.

We note that this also proves that $\widehat{G}$ is weakly Hausdorff.

The reduction of the Diestel-Kühn theory to ours is completed by showing that the two cycle spaces coincide, i.e., proving Theorem 30. One simple way to see this is as follows. If $T$ is a pre-tree of $\widetilde{G}$ (pre-trees are discussed on pp. 856-857 of [10]), then there is a minimal connected spanning set of $\widehat{G}$ with the same edges as $T$. It is easy to see that the fundamental cycles are the same in the two cases. Since these fundamental cycles generate the two cycle spaces algebraically in the same way, the two cycle spaces coincide.

Diestel and Kühn give an interesting example. It is shown here in Figure 1, which is essentially Figure 5 from [10]. (The line segment through all the vertices from $x$ to $y$ is not part of the graph, except for the dyadic points, which are the vertices of the graph.) This graph is constructed as follows. We start with two vertices $x$ and $y$, joined by an edge. At each iteration of its construction, each consecutive pair of vertices has a new vertex placed between them and this is made adjacent to both members of the consecutive pair. This graph has a set $\mathcal{P}$ of infinitely many edge-disjoint paths joining the vertices $x$ and $y$. No two vertices are joined by infinitely many internally-disjoint paths. We may take one member of $\mathcal{P}$ to be the edge joining $x$ and $y$. 


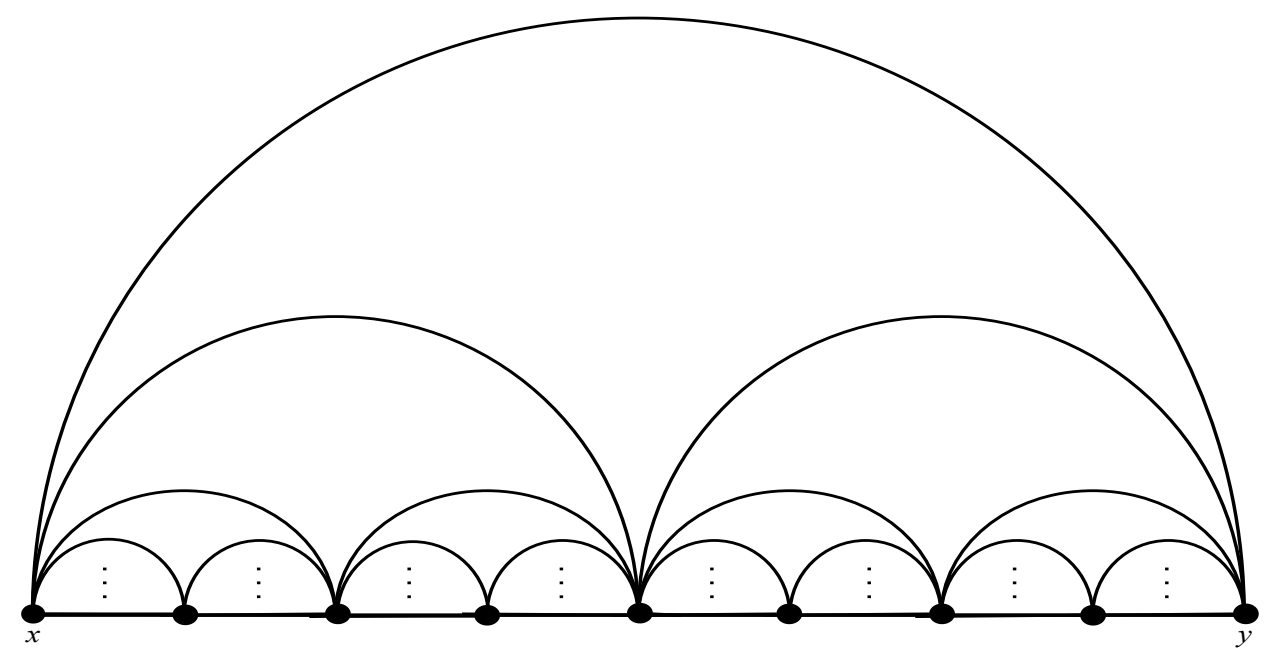

FIGURE 1

If $Q, R \in \mathcal{P}$, then $Q \cup R$ is the edge-disjoint union of finitely many finite cycles in $G$ and, therefore, $E(Q \cup R)$ is in the cycle space. By letting $P=P_{1}, P_{2}, \ldots$ be an infinite sequence of distinct paths in $\mathcal{P}$, we see that $E(P)$ is the symmetric difference of $E\left(P_{1} \cup P_{2}\right), E\left(P_{2} \cup P_{3}\right), E\left(P_{3} \cup P_{4}\right), \ldots$ Thus $E(P)$ is in the cycle space.

However, this is not the only reason $E(P)$ is in the cycle space. In fact, it is a cycle of $\widetilde{G}$ ! It is not completely obvious, but the set of non-edges of $\widetilde{G}$ is homeomorphic to $[0,1]$, with $x$ and $y$ corresponding to 0 and 1 . (This is the line segment joining $x$ and $y$ in Figure 1.) Each vertex dominates precisely two rays, except $x$ and $y$ dominate one each. Thus $P \cup V$ has the property that deleting any one edge will not disconnect this set, but, vacuously, deleting any two edges will. The ends are "hiding" the fact that the connection in the space is much richer than provided for by just considering the edges. This is where the "extra" cycles come from - even extra finite cycles.

By Theorem 27, the cycle space is the same as for the space obtained by contracting the one component of vertices and ends to a single point. In particular, in this contracted space, every edge is a loop and so obviously is in the cycle space.

In general, any circle $C$ in $\widetilde{G}$ is a cycle and, being a compact subset of a Hausdorff space, is necessarily closed. Conversely, it is not hard to show (using the fact that every closed subset of $\widetilde{G}$ is arcwise connected [10, Thm. 5.3]) that if $C$ is a closed cycle in $\widetilde{G}$, then there is a circle $C^{\prime}$ in $\widetilde{G}$ so that $E \cap C^{\prime}=E \cap C$.

In Figure 2, the line segment $L$ between $x$ and $z$ consists of nonedge points of the weakly Hausdorff space $X$; the solid segments are the edges. The set of all edges is an edge cycle, but there is no closed cycle containing all the edges. Thus, although the cycle spaces are the same for finitely connected $G$, our cycles are not guaranteed to correspond to circles. We shall however establish that 


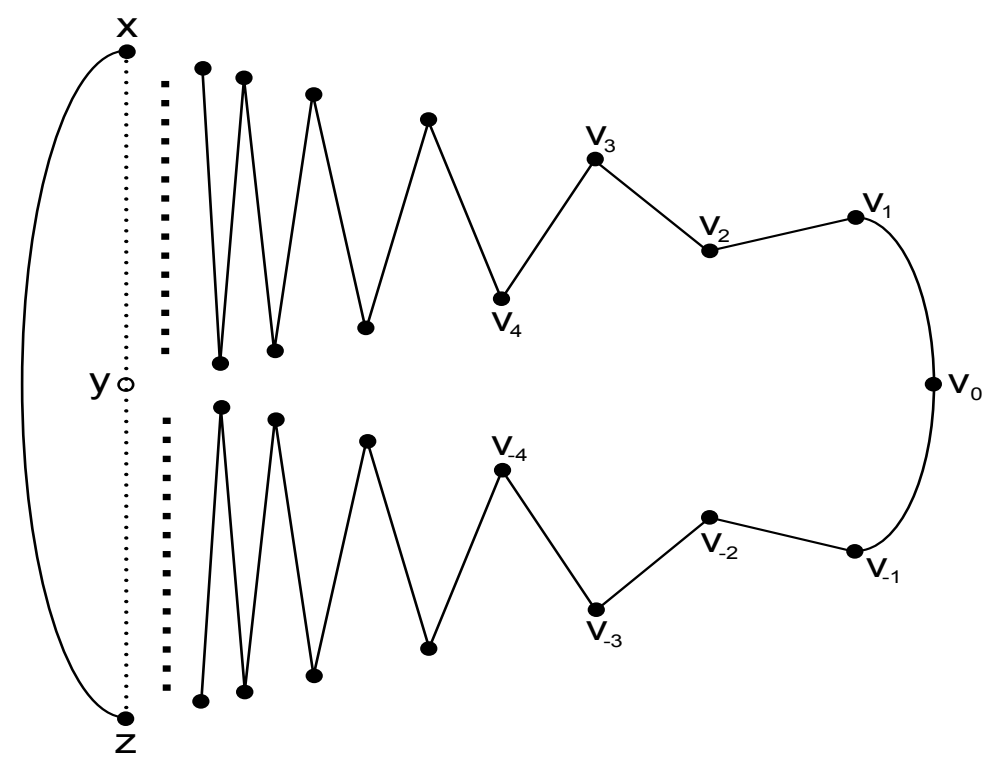

Figure 2. A non-closed cycle

this correspondence does occur if $G$ is a finitely edge-connected graph (no two vertices are joined by infinitely many edge-disjoint paths). Diestel and Kühn consider such graphs in order to guarantee the existence of topological spanning trees [10, Thm. 5.2].

The following result shows the a cycle that is not closed can only occur if some of the components of $X \backslash E$ are not trivial. Our proof relies on a recent result of Thomassen and Vella [18, Cor. 2.3], which requires the additional assumption that $X$ is metric. For our purposes, this is not a major addition, since, for example, we shall see in the next section that $\widetilde{G}$ is metric when $G$ is finitely connected.

Theorem 35. Let $(X, E)$ be a compact, connected, metric generalized edge space such that every edge is a 1-cell. If $X-E$ is totally disconnected, then a subset of $X$ is a cycle if and only if it is a circle. In particular, all cycles are closed.

Proof: Let $C$ be a cycle in $X$ and let $e_{0}$ be an edge of $C$ with ends $a, b$. If $e_{0}$ is a loop, then $C=\mathrm{Cl}\left(e_{0}\right)$ is a circle. Hence we may assume that $a \neq b$. It suffices to prove that $P=C \backslash e_{0}$ is an arc (a homeomorph of $[0,1]$ ). The proof consists of showing $P$ is an $a b$-path in the following sense: if $a$ and $b$ are the ends of $e_{0}$, then $P$ is a connected set containing $a$ and $b$ and, for every other point $x$ of $P, a$ and $b$ are in different components of $P \backslash x$. By the comment following Corollary 2.3 in [18], $P$ is locally connected. (A compact totally disconnected space, such as $X-E$, is 0 -dimensional [4, Thm. 4.A.11], so $(X, E)$ is a graph-like space as 
defined in [18].) By Theorem 2.3.17 of [19], $P$ is compact (the $a b$-prepaths in [19] are the $a b$-paths as defined here). Since $X$ is metric, $P$ is an arc [20, Thm. 28.13]. Moreover, since $C$ is compact, $C$ is closed.

So it suffices to show that $P$ is an $a b$-path. It is clear that if $e$ is an edge of $P$, then $P \backslash e$ has precisely two components $K_{a, e}$ and $K_{b, e}$, the former containing $a$ and the latter containing $b$.

The main point in the proof is to show that if $x, y \in P-E$, then there is an edge $e$ of $P$ so that $x$ and $y$ are in different components of $P \backslash e$. So suppose there is an $x \in P-E$ so that some other $y \in P-E$ is such that, for every $e \in E, x$ and $y$ are in the same component of $P \backslash e$. Since $X$ is Hausdorff and $P$ is locally connected, $x$ and $y$ have disjoint connected open neighbourhoods $U_{x}$ and $U_{y}$, respectively.

Since $X-E$ is 0 -dimensional and $P$ is connected, the edges of $P$ are dense in $P$. That is, there is an edge $e$ of $P$ such that $e \cap U_{x} \neq \emptyset$. Since $U_{x}$ is connected, some end of $e$ is in $U_{x}$. The component $K^{e}$ of $P \backslash e$ containing $x$ also contains $y$. Thus, $e \cap U_{y}=\emptyset$, so $U_{y}$ is contained in $K^{e}$. Then $P \backslash K^{e}$ is an open subset of $P$ containing $e$, and we set

$$
U_{x}^{\prime}=U_{x} \cup\left(\bigcup_{e \cap U_{x} \neq \emptyset}\left(P \backslash K^{e}\right)\right) .
$$

This is a connected open set containing $x$ and disjoint from $U_{y}$.

We claim $U_{x}^{\prime}$ contains precisely one of $a$ and $b$. Let $e^{*}$ be a fixed edge intersecting $U_{y}$ and let $K^{*}$ be the component of $P \backslash e^{*}$ not containing $x$ and $y$. Then $K^{*} \cup e^{*} \cup U_{y}$ is a connected set disjoint from $U_{x}$ and contains precisely one of $a$ and $b$. Now if $e$ is any edge so that $e \cap U_{x} \neq \emptyset$, then $P \backslash e$ has a component $K$ containing $x, y$, and precisely one of $a$ and $b$. Since $K^{*} \cup e^{*} \cup U_{y}$ is connected and disjoint from $e$, it is contained in $K$, so $K$ contains the one of $a$ and $b$ in $K^{*}$.

It follows that $U_{x}^{\prime}$ is a connected open set in $P$, disjoint from $U_{y}$, and contains one of $a$ and $b$. Likewise, we can construct $U_{y}^{\prime}$, containing $U_{y}$, disjoint from $U_{x}^{\prime}$, and containing the other of $a$ and $b$. Thus there is no other $z \in P-E$ not separated from $x$ in $P$ by some $e \in E$ (only one of $U_{x}^{\prime}, U_{y}^{\prime}, U_{z}^{\prime}$ contains $a$, only one contains $b$, and each contains at least one of $a$ and $b$ ).

We aim for a contradiction by showing that $P=U_{x}^{\prime} \cup U_{y}^{\prime}$, since then $U_{x}^{\prime}, U_{y}^{\prime}$ is a separation of $P$, showing $P$ is not connected. It is clear that if $e$ is an edge and $e \cap\left(U_{x}^{\prime} \cup U_{y}^{\prime}\right) \neq \emptyset$, then $e \subseteq U_{x}^{\prime} \cup U_{y}^{\prime}$. So suppose there is an edge $e$ so that $e \nsubseteq U_{x}^{\prime} \cup U_{y}^{\prime}$. Then $e \cap\left(U_{x}^{\prime} \cup U_{y}^{\prime}\right)=\emptyset$ so $U_{x}^{\prime}$ and $U_{y}^{\prime}$ are each in a single component of $P \backslash e$. Since $x$ and $y$ are in the same component of $P \backslash e$ by assumption, $U_{x}^{\prime} \cup U_{y}^{\prime}$ is in the same component of $P \backslash e$, putting $a$ and $b$ in the same component of $P \backslash e$, a contradiction. Thus, every edge of $P$ is in $U_{x}^{\prime} \cup U_{y}^{\prime}$.

It remains to show that if $z$ is a vertex other than $x$ or $y$, then $z \in U_{x}^{\prime} \cup U_{y}^{\prime}$. Choose the labelling so that $a \in U_{x}^{\prime}$ and $b \in U_{y}^{\prime}$. There is an edge $e$ such that $z$ is in one component $K$ of $P \backslash e$ and $x$ and $y$ are in the other component. For sake of definiteness, we assume $e \subseteq U_{x}^{\prime}$. Then $e \subseteq P \backslash K^{e^{\prime}}$, for some edge $e^{\prime}$ 
such that $e^{\prime} \cap U_{x} \neq \emptyset$. Now $U_{y}^{\prime} \subseteq K^{e^{\prime}}$, so $b \in K^{e^{\prime}}$ and, therefore, $a \in P \backslash K^{e^{\prime}}$. Consequently, $K \subseteq P \backslash K^{e^{\prime}}$, so $z \in U_{x}^{\prime}$.

Thus, any two points of $P-E$ are separated by some edge. For $x \in P-E, x \notin$ $\{a, b\}$, let $E_{a}$ be the set of edges $e$ such that $x$ is not in the component $K^{e}$ of $P \backslash e$ containing $a$ and let $E_{b}$ be the set of edges $e$ such that $x$ is not in the component $K^{e}$ of $P \backslash e$ containing $b$. Clearly $E=E_{a} \cup E_{b}$ and $P \backslash x=\bigcup_{e \in E}\left(K^{e} \cup e\right)$. The latter shows that $a$ and $b$ are in different components of $P \backslash x$, as required.

When $G$ is finitely edge-connected, we aim to use Theorem 35 to show that cycles are circles in $\widetilde{G}$. Theorem 28 shows that each block of $G$ is compact. In the next section, we show - even when $G$ is only finitely connected - that $\widetilde{G}$ is metric. Here we show the remaining hypothesis of Theorem 35 holds.

Lemma 36. If $G$ is a finitely edge-connected graph, then $\widetilde{G}-E$ is 0-dimensional.

Proof: Given arbitrary $p_{1}, p_{2} \in \widetilde{G}-E$, we require a separation of $\widetilde{G}-E$ with $p_{1}, p_{2}$ on different sides. It is sufficient to find a finite set $F$ of edges in $\mathbf{F}(G)$ such that the equivalence classes $p_{1}, p_{2}$ of $\mathbf{F}(G)$ belong to distinct components of $\mathbf{F}(G)-F$. All such components are necessarily saturated as well as closed, so that their images are closed in $\widetilde{G}$. Note moreover that the (finitely many) components of $\mathbf{F}(G)-F$ are simply the closures of the components of $G-F$.

Since no end of $G$ is dominated by more than one vertex, each equivalence class in $\mathbf{F}(G)$ either consists of an undominated end, or else has a unique vertex representative. Let $p_{i}^{\prime}$ be a representative of $p_{i}$, chosen to be a vertex if possible, and suppose first that both $p_{1}^{\prime}$ and $p_{2}^{\prime}$ are vertices. Since they are not joined by infinitely many edge-disjoint paths in $G$, they are separated in $G$ by a finite set $F$ of edges. Suppose next that $p_{1}^{\prime}$ is a vertex but $p_{2}^{\prime}$ is an end. Since the vertex $p_{1}^{\prime}$ does not dominate the end $p_{2}^{\prime}$, there exists a finite set $S$ of vertices separating $p_{1}^{\prime}$ from $p_{2}^{\prime}$ in $G$. From the preceding remarks, each of the vertices in $S$ can be separated in $G$ from $p_{1}^{\prime}$ by a finite edge cut, and we may take $F$ to be the union of these finite edge cuts. Finally, suppose $p_{1}^{\prime}, p_{2}^{\prime}$ are both undominated ends. Then they are separated by a finite set $S$ of vertices. For any $v \in S$, since $v$ does not dominate $p_{1}^{\prime}$, by the previous argument $v$ can be separated in $G$ from $p_{1}$ by a finite set of edges $T_{v}$, and we may take for $F$ the union of $T_{v}$ over $v \in S$.

\section{Aside: $\widetilde{G}$ IS A PEANO SPACE}

In [16], Sprussel asks: is $\widetilde{G}$ a metric space? We prove the following more general fact.

Theorem 37. If $G$ is a 2-connected, finitely connected graph, then $\widetilde{G}$ is a Peano space.

We remind the reader that a Peano space is a compact, connected, locally connected, metric space. We are fortunate here to have already proved that $\widetilde{G}$ is compact (Corollary 34) and Hausdorff (Theorem 29). It is obviously connected 
and, being a quotient of the locally connected space $\mathbf{F}(G)$, it is locally connected. A standard theorem says a compact space is metric if and only if it is Hausdorff and second countable [13, Ch. 4, Thm. 16]. (A topological space is secondcountable if its topology has a countable base.) Note that Lemma 32 implies that $\widetilde{G}$ is second-countable. A Peano space is always arcwise connected.

The following is a useful result [13, Ch. 5, Thm. 20].

Lemma 38. If $\mathcal{P}$ is an upper semicontinuous partition of a second countable space $X$ so that the members of $\mathcal{P}$ are compact, then the quotient space with pointset $\mathcal{P}$ is second countable.

For us, this means that we need only show that the sets in $\mathbf{F}(G)$ consisting of a vertex and its dominated ends are closed (since $\mathbf{F}(G)$ is compact by Corollary 34 and a closed subset of a compact set is compact) and that the partition into these closed sets is upper semicontinuous. The first is done in [10], being the easy Lemma 4.1.

For the remainder of this section, $\mathcal{P}$ is the partition of $\mathbf{F}(G)$ in which each part is either a vertex and all ends it dominates or a singleton from an edge, or a singleton consisting of an undominated end.

Lemma 39. Let $G$ be a finitely connected graph and let $S$ be a finite subset of $V(G)$. For each $s \in S$, let $E_{s}$ be the part in $\mathcal{P}$ containing s. Let $K$ be any topological component of $\mathbf{F}(G) \backslash S$. Then $K \backslash\left(\cup_{s \in S} E_{s}\right)$ is open and saturated in $\mathbf{F}(G)$.

Proof. We have that $K$ is open by definition. As mentioned above, from [10] we know each $E_{s}$ is closed. Since $S$ is finite, we see that $K \backslash\left(\cup_{s \in S} E_{s}\right)$ is open.

To see that $K \backslash\left(\cup_{s \in S} E_{s}\right)$ is saturated, let $E$ be any part such that $E \cap(K \backslash$ $\left.\cup_{s \in S} E_{s}\right) \neq \emptyset$. If $E$ is degenerate, then it is trivial that $E \subseteq K \backslash\left(\cup_{s \in S} E_{s}\right)$. Otherwise, let $v$ be the unique vertex in $E$. If $v \notin S$, then $v$ and any end it dominates must be in the same component of $G-S$. Hence $E \subseteq K \backslash\left(\cup_{s \in S} E_{s}\right)$. If $v \in S$, then $E=E_{v}$ and $E \cap\left(K \backslash\left(\cup_{s \in S} E_{s}\right)\right)=\emptyset$.

Lemma 40. Let $G$ be a finitely connected graph. Then $\mathcal{P}$ is an upper semicontinuous decomposition.

Proof. Let $U$ be open in $\mathbf{F}(G)$ and let $E$ be a part contained in $U$. When $E$ is a point in the interior of an edge, that there is a saturated open set $V$ containing $E$ and contained in $U$ is trivial. If $E$ is just a vertex, then some basic neighbourhood of $v$ is contained in $U$ and all such neighbourhoods are saturated.

So we can assume $E$ contains an end $\omega$. Since $U$ is open, there is some finite set $S$ of vertices such that the component $K$ of $\mathbf{F}(G)-S$ containing $\omega$ is contained in $U$. If $\omega$ is not dominated by any vertex in $S$, then Lemma 39 gives the desired saturated open subset of $U$. If $\omega$ is dominated by a vertex $v \in S$, then $U$ contains a basic neighbourhood $N$ of $v$. In this case, $N \cup\left(K \backslash\left(\cup_{s \in S \backslash\{v\}} E_{s}\right)\right)$ is open, saturated, and contained in $U$.

As mentioned just before Lemma 36, we now have the following. 
Corollary 41. If $G$ is a finitely-edge-connected graph, then a subset of $\widetilde{G}$ is a cycle if and only of it is a circle.

Lemma 36, Theorem 37, and [18, Cor. 2.3$]$ imply that $\widetilde{G}$ is hereditarily locally connected. This in turn implies [10, Thm. 5.3]: if $G$ is finitely edge-connected, then every closed connected subset of $\widetilde{G}$ is arcwise connected.

\section{Remarks about the Bonnington-Richter Cycle Space}

If $G$ is a locally finite graph, then the Alexandroff compactification $\mathbf{A}(G)$ of $G$ is obtained from $\mathbf{F}(G)$ by identifying all the points in $\mathbf{F}(G) \backslash G$ to a single point. It is easy to see that $\mathbf{A}(G)$ is compact and that the edge set of any connected 2-regular subgraph is an edge cycle. Thus, the cycle space of $\mathbf{A}(G)$ is precisely the one introduced in [1].

In that work, an embedding of a locally finite graph $G$ in the sphere is considered. If there are only finitely many accumulation points, then the closure of $G$ in the sphere is a compactification $K$ of $G$ and also has a cycle space, which is contained in the cycle space of $\mathbf{A}(G)$. A main result of [1] is finding a basis for the quotient of these two cycle spaces. This very naturally leads one to wonder about the relationships between the cycle spaces of different compactifications of a locally finite graph $G$. This work is begun in [3], where it is shown that if $(X, E)$ and $(Y, F)$ are compact weakly Hausdorff edge spaces and $f: X \rightarrow Y$ is a continuous function such that $f: E \rightarrow F$ is a bijection and $f:(X \backslash E) \rightarrow(Y \backslash F)$, then, identifying $e$ and $f(e)$, the cycle space of $(X, E)$ is contained in that of $(Y, F)$. Still open is the general question of how to find a basis for the quotient space.

\section{Feebly Hausdorff Spaces}

In the context of edge spaces, any open set containing a vertex must also contain all the edges incident with that vertex. Thus, if we are trying to separate two vertices $u$ and $v$ by open sets, the intersection must contain all the edges incident with both $u$ and $v$. In the preceding sections, we were required, in particular, to forbid infinite sets of parallel edges between two vertices - their basic neighbourhoods have infinitely many edges in common. It is the purpose of this section to show how to relax the "weakly Hausdorff" condition to allow more general situations, including infinitely many parallel edges.

Let $X$ be a topological space and let $v \in X$. We set $v^{\diamond}$ to be the intersection of all the open sets containing $v$ (so a topological space is Alexandroff discrete if and only if, for every point $x$, the set $x^{\diamond}$ is open). The space $X$ is feebly Hausdorff if, for any two points $u, v \in X$, there exist open sets $U_{u}$ and $U_{v}$ containing $u$ and $v$, respectively, such that $U_{u} \cap U_{v} \subseteq u^{\diamond} \cap v^{\diamond}$. That is, there are open sets which intersect just in those points that they must contain, as the intersection of any pair of neighbourhoods contains these points. (Thus, we could replace $\subseteq$ by $=$.) 
A simple example of a feebly Hausdorff space which is not weakly Hausdorff is given by the graph with two vertices joined by infinitely many edges, equipped with the classical topology. Note that this space is compact, but replacing the edges with arcs, to obtain a Hausdorff space, gives a non-compact topology.

It turns out, and the theory is developed fully in [19], that we can extend many of our arguments to the case of connected, compact feebly Hausdorff edge spaces $(X, E)$. The proofs are typically quite a bit more involved and the statements are not so clean. In particular, in this context we need to define the bond space to be the weak span of the finite bonds (equivalently, the space of all finite edge cuts). Note that, for weakly Hausdorff spaces, this definition is equivalent to the one we used previously. Here we only wish to indicate the flavour of the theory.

Firstly, there still exist minimal connected spanning subsets and the appropriate analogue of Lemma 3 holds, so that we may obtain, as before, fundamental cycles $C_{e}$, with respect to any minimal connected spanning set. Secondly, it is still true that the fundamental cycles strongly generate the cycle space.

In order to deal with orthogonality, the main tool used is to express the cycle space of a compact feebly Hausdorff edge space $(X, E)$ in terms of that of a certain quotient $(Y, F)$. In this case, we construct an intermediate edge space $(X, F)$, where $F \subseteq E$ consists precisely of the edges whose parallel class is finite, that is, edges which do not share two ends with infinitely many other edges. The edge space $(Y, F)$ is the simplification (as in Section 5$)$ of $(X, F)$ (since edges are points, we do not distinguish between the edges of $(X, F)$ and their formally distinct images in the quotient). We show that $(Y, F)$ is weakly Hausdorff, and that the bond spaces in all three edge spaces are the same. Moreover, the edge sets of the minimal spanning sets, and the corresponding fundamental cycles, are precisely the same in $(X, F)$ and $(Y, F)$. Hence, as a corollary of the fact that the fundamental cycles strongly generate the cycle space, we have that the corresponding cycle spaces are isomorphic (as in Section 5, although the proof is different). In order to bridge the gap between the cycle spaces of $(X, E)$ and $(X, F)$, we take a minimal spanning set of $T$ of $(X, F)$, which we then consider as an edge subspace $T^{\prime}:=(T, T \cap E)$ of $(X, E)$. Finally we use a minimal spanning set of $T^{\prime}$ to extend the fundamental cycles of $(X, F)$ to fundamental cycles of $(X, E)$.

Theorem 42. ([19], Thm. 5.4.6) Let $(X, E)$ be a connected compact feebly Hausdorff edge space. With $Y$ and $F$ as in the preceding paragraph, the cycle space of $(X, E)$ is $\mathcal{Z}(Y, F) \oplus 2^{E \backslash F}$. For any minimal connected spanning set of $(X, E)$, the set $\mathcal{F}$ of fundamental cycles is such that $\mathcal{A}^{2}(\mathcal{F})$ is the cycle space of $(X, E)$.

We remark that, as can be seen in the above discussion, the arguments depend heavily on the fact that we are free to declare what the edges are, that is, we need not include all the open singletons in the edge set of an edge space.

Theorem 43. ([19, Thm. 5.4.8]) Let $(X, E)$ be a connected compact feebly Hausdorff edge space, with cycle space $\mathcal{Z}(X, E)$ and bond space $\mathcal{B}(X, E)$. Then 
$\mathcal{B}(X, E)^{\perp}=\mathcal{Z}(X, E)$ and, letting $E^{\prime}=\{e \in E \mid X \backslash e$ is connected $\}, \mathcal{Z}(X, E)^{\perp}=$ $\mathcal{B}\left(X, E^{\prime}\right) \oplus 2^{E \backslash E^{\prime}}$. In particular, if $(X, E)$ is 2-edge-connected, then $\mathcal{Z}(X, E)^{\perp}=$ $\mathcal{B}(X, E)$.

Again, the flexibility to ignore the edges in $E \backslash E^{\prime}$ makes for a cleaner statement and simplifies the arguments.

\section{ACKnowledgment}

We are grateful to the referees for their thoughtful comments.

\section{REFERENCES}

[1] C.P. Bonnington and R.B. Richter, Graphs embedded in the plane with a bounded number of accumulation points. J. Graph Theory 44 (2003), no. 2, 132-147.

[2] H. Bruhn, The cycle space of a 3-connected locally finite graph is generated by its finite and infinite peripheral circuits. J. Combin. Theory Ser. B 92 (2004), no. 2, 235-256.

[3] K. Casteels and R.B. Richter, The cycle spaces of an infinite graph, in preparation.

[4] C.O. Christenson and W.L. Voxman, Aspects of Topology, B.C.S. Associates, Moscow, Idaho, 1998.

[5] R. Diestel, End spaces and spanning trees, J. Combin. Theory Ser. B 96 (2006), 846-854.

[6] R. Diestel, Graph Theory, Number 173 in Graduate Texts in Mathematics. Springer Verlag, New York, 2000.

[7] R. Diestel, The cycle space of an infinite graph, Comb. Probab. Comput. 14 (2005), 59-79.

[8] R. Diestel and D. Kühn, On infinite cycles I, Combinatorica 24 (2004), 68-89.

[9] R. Diestel and D. Kühn, On infinite cycles II, Combinatorica 24 (2004), 91-116.

[10] R. Diestel and D. Kühn, Topological paths, cycles and spanning trees in infinite graphs, Europ. J. Combin. 25 (2004), 835-862.

[11] R. Diestel and D. Kühn, Graph theoretical versus topological ends of graphs, J. Combin. Theory Ser. B 87 (2003), 197-206.

[12] K. L. Hofmann, The low separation axioms $T_{0}$ and $T_{1}$, in Encyclopedia of General Topology, 155-157. Elsevier Science B.V., Amsterdam, 2004.

[13] J.L. Kelley, General Topology, Springer-Verlag, New York-Heidelberg-Berlin, 1975.

[14] K. Kuratowski, Topology, Vol. II, New edition (A. Kirkor, trans.), Academic Press, New York, 1968.

[15] J. Nikiel, H.M. Tuncali and E.D. Tymchatyn, Continuous images of arcs and inverse limit methods. Mem. Amer. Math. Soc., 104 (1993), no. 498, viii +80.

[16] P. Sprussel, End spaces of graphs are normal, preprint, 2006.

[17] L.A. Steen and J.A. Seebach, Counterexamples in Topology, Holt, Rinehart and Winston, New York, 1970.

[18] C. Thomassen and A. Vella, Graph-like continua, augmenting arcs, and Menger's Theorem, submitted.

[19] A. Vella, A Fundamentally Topological Perspective on Graph Theory, Ph.D. thesis, U. Waterloo, 2005. Available at http://etd.uwaterloo.ca/etd/avella2005.pdf

[20] S. Willard, General Topology, Addison-Wesley, Reading, 1970. 